\title{
The Episodic, Precessing Giant Molecular Outflow from IRAS 04239+2436 (HH 300)
}

\section{Citation}

Arce, Hector G., and Alyssa A. Goodman. 2001. “The Episodic, Precessing Giant Molecular Outflow from IRAS 04239+2436 (HH 300)." The Astrophysical Journal 554 (1): 132-51. https:// doi.org/10.1086/321334.

\section{Permanent link}

http://nrs.harvard.edu/urn-3:HUL.InstRepos:41397468

\section{Terms of Use}

This article was downloaded from Harvard University's DASH repository, and is made available under the terms and conditions applicable to Other Posted Material, as set forth at http:// nrs.harvard.edu/urn-3:HUL.InstRepos:dash.current.terms-of-use\#LAA

\section{Share Your Story}

The Harvard community has made this article openly available.

Please share how this access benefits you. Submit a story. 
The Astrophysical Journal, 554:132-151, 2001 June 10

(C) 2001. The American Astronomical Society. All rights reserved. Printed in U.S.A.

\title{
THE EPISODIC, PRECESSING GIANT MOLECULAR OUTFLOW FROM IRAS 04239+2436 (HH 300)
}

HéCtor G. Arce and Alyssa A. Goodman

Harvard-Smithsonian Center for Astrophysics, 60 Garden Street, Cambridge, MA 02138; harce@cfa.harvard.edu, agoodman@cfa.harvard.edu Received 2000 October 17; accepted 2001 February 1

\begin{abstract}
We present the first set of detailed molecular-line maps of the region associated with the giant HerbigHaro flow HH 300 from the young star IRAS $04239+2436$. Our results indicate that the red lobe of the HH 300 flow is depositing a fair amount of momentum [3.2(sin $\left.i)^{-1} M_{\odot} \mathrm{km} \mathrm{s}^{-1}\right]$ and kinetic energy $\left[2.6(\sin i)^{-2} \times 10^{43}\right.$ ergs $]$ over a notable volume $(\sim 11 \%)$ of its host dark cloud. This makes HH 300 a key player in the evolution and fate of its parent cloud. The redshifted molecular outflow lobe of $\mathrm{HH}$ 300 is $1.1 \mathrm{pc}$ long and $0.3 \mathrm{pc}$ wide and has a very clumpy structure. The density, velocity, and momentum distributions in the outflow indicate that the observed clumps arise from the prompt entrainment of ambient gas. Bow shock-shaped structures are associated with the clumps, and we hypothesize that the shocks are produced by different mass ejection episodes. Lines drawn from IRAS $04239+2436$ to each of these clumps have different orientations on the plane of the sky, and we conclude that $\mathrm{HH} 300$ is a precessing and episodic outflow. The observations include a map of the red lobe in the CO (2-1) line, with a beam size of $27^{\prime \prime}$, and more extended maps of the outflow region in the ${ }^{12} \mathrm{CO}(1-0)$ and ${ }^{13} \mathrm{CO}$ (1-0) lines, with $45^{\prime \prime}$ and 47 " beam sizes, respectively. Due to "contamination" by emission from another molecular cloud along the same line of sight, we are not able to study the blueshifted lobe of HH 300 . The combined ${ }^{12} \mathrm{CO}(1-0)$ and ${ }^{13} \mathrm{CO}(1-0)$ line observations enable us to estimate the outflow mass accounting for the velocity-dependent opacity of the ${ }^{12} \mathrm{CO}(1-0)$ line. This method is much more precise than using ${ }^{12} \mathrm{CO}$ data alone. We obtain a steep power-law mass spectrum for $\mathrm{HH} 300$, which we believe is best explained by the evolution of the outflow mass kinematics. In addition, our ${ }^{13} \mathrm{CO}(1-0)$ observations show that the $\mathrm{HH} 300$ flow has been able to redistribute (in space and velocity) considerable amounts of its surrounding medium-density $\left(\sim 10^{3} \mathrm{~cm}^{-3}\right)$ gas.
\end{abstract}

Subject headings: ISM: clouds - ISM: Herbig-Haro objects — ISM: individual (B18, HH 300) ISM: jets and outflows - stars: formation

\section{INTRODUCTION}

A Herbig-Haro (HH) object is a nebulous knot that delineates the shock arising from the interaction of a highvelocity flow of gas ejected by a young stellar object (YSO) and the ambient medium. A chain of these $\mathrm{HH}$ objects (or knots) is usually referred as an HH flow. Recently, it has been found that very long (parsec scale) HH flows exist, and that they are quite common (Reipurth, Bally, \& Devine 1997, hereafter RBD). This has sparked major interest on the impact of these energetic mass flows on their environment, in particular, the relation between optical jets and molecular outflows and the physics of the entrainment mechanism. Giant HH flows have sizes about an order of magnitude larger than the cloud cores from which they originate, and many are found to extend to distances well outside the boundary of their parent dark cloud (RBD). One or more shocks associated with the same flow can accelerate the entrained gas to velocities greater than those of the quiescent cloud, transferring momentum and energy into the molecular cloud and thus producing a molecular outflow. The colossal size of a giant $\mathrm{HH}$ flow enables it to entrain molecular material at parsec-scale distances from the source, and thus it may affect the kinematics and density of a substantial volume of its parent molecular cloud.

Although several models try to explain the entrainment mechanism that forms molecular outflows (e.g., Shu et al. 1991; Masson \& Chernin 1993) and numerous other theoretical works investigate the effects YSO outflows have on their environment (e.g., Norman \& Silk 1980; Raga, Cantó, \& Steffen 1996; Matzner \& McKee 2000), there has yet to be a consensus reached on these issues. On the observa- tional front, recent high-resolution studies of molecular outflows (e.g., Bence, Richer, \& Padman 1996; Cernicharo \& Reipurth 1996; Lada \& Fich 1996; Davis et al. 1997; Yu, Billawala, \& Bally 1999; Lee et al. 2000) have provided significant new constraints on the physical parameters of outflows and the entrainment mechanism.

In order to study the effects an outflow has on its environment's kinetic and density structure, it is imperative to observe a large area of the cloud gas surrounding the outflow, in addition to the outflow itself. The observations should preferably be of more than one molecular-line transition, probing a range of densities, and at least one of the lines should be relatively optically thin in order to take line opacity into account. In this paper, we present new ${ }^{12} \mathrm{CO}$ $(2-1),{ }^{12} \mathrm{CO}(1-0)$, and ${ }^{13} \mathrm{CO}(1-0)$ observations of the $\mathrm{HH}$ 300 outflow, its surroundings, and a large fraction of its parent dark cloud.

The HH 300 flow was discovered, in the optical, by RBD. $\mathrm{HH} 300$ is located in the westernmost region of the B18 molecular cloud (usually called B18w) in the Taurus cloud complex. The B18w region, also called IRAS core Tau G1 by Wood, Myers, \& Daugherty (1994), looks like a 1.7 pc $\times 0.7$ pc filamentary protuberance from the main cloud (B18; see Fig. 1). The source of the HH 300 flow was established to be IRAS $04239+2436^{1}$ (RBD), which lies at the northern end of $\mathrm{B} 18 \mathrm{w}$, just west of the "bridge" between B18 and B18w. Near- and mid-infrared photometry (Myers

\footnotetext{
${ }^{1}$ Although this paper is the first one to present a map of the HH 300 molecular outflow, Moriarty-Schieven et al. (1992) observed high-velocity wings in the ${ }^{12} \mathrm{CO}(3-2)$ line at the source position, which led them to conclude that IRAS $04239+2436$ is a molecular outflow source.
} 


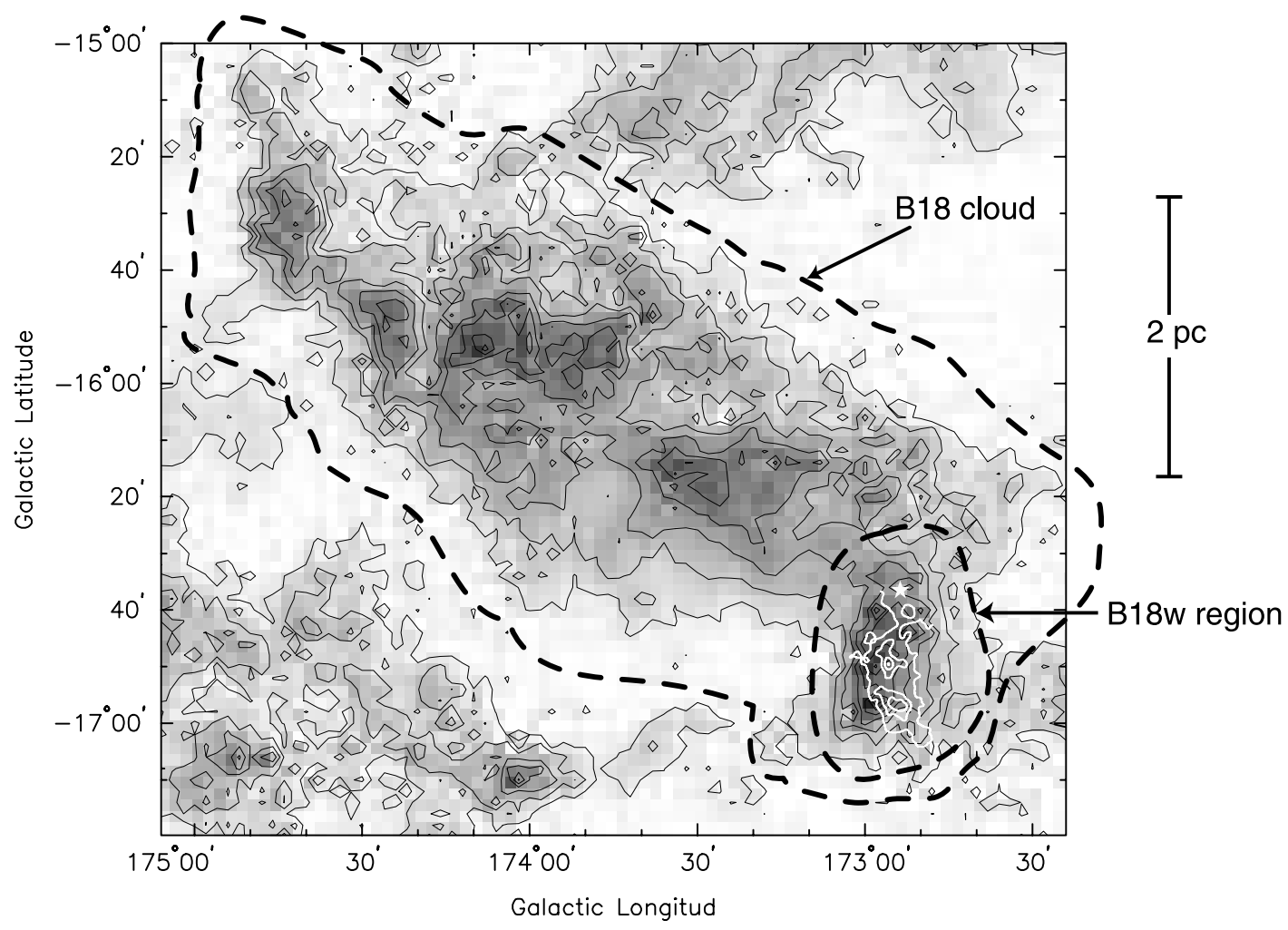

FIG. 1.-The ${ }^{13} \mathrm{CO}(1-0)$ integrated intensity map of the B18 cloud in Taurus for the velocity range $2.9 \mathrm{~km} \mathrm{~s}^{-1}<v<8.9 \mathrm{~km} \mathrm{~s}^{-1}$. The map is part of the large-scale ${ }^{13} \mathrm{CO}(1-0)$ map of the Taurus molecular cloud complex from Mizuno et al. (1995). The source of the HH 300 flow, IRAS 04239+2436, is shown with a white star symbol. The integrated intensity map of the HH 300 molecular outflow's red lobe is shown in white contours. The B18 and B18w regions are identified.

et al. 1987) and its infrared spectrum (Greene \& Lada 1996) indicate that this is a $1.3 L_{\odot}$ class I young stellar object. The HH 300 flow was originally discovered to consist of three redshifted $\mathrm{HH}$ objects (HH 300A, 300B, and 300C), each with a bow shock-like morphology, close together at a distance of about $1.1 \mathrm{pc}$ southwest of the outflow source, and a small blueshifted knot (HH 300D) about 0.02 pc northeast of the source, assuming a distance to Taurus of $140 \mathrm{pc}$ (Kenyon, Dobrzycka, \& Hartmann 1994). Recent nearinfrared camera multiobject spectrograph images at 1.644 and $2.122 \mu \mathrm{m}$, with a spatial resolution of $\sim 0$ ". 13 , of the $\mathrm{HH}$ 300 source region by Reipurth et al. (2000) reveal a cometary nebula surrounding the source, a jet on the blueshifted side of the HH flow (northeast of the source) along the symmetry axis of the nebula, and that IRAS $04239+2436$ is a binary.

\section{OBSERVATIONS}

\section{1. ${ }^{12} \mathrm{CO}(2-1)$ Data}

The ${ }^{12} \mathrm{CO}(2-1)$ line data were obtained using the on-thefly mapping technique at the National Radio Astronomy Observatory (NRAO) $12 \mathrm{~m}$ telescope on Kitt Peak, Arizona, in 1998 December. At the observed frequency of $230 \mathrm{GHz}$, the telescope's half-power beamwidth, main beam efficiency, and aperture efficiency are $27^{\prime \prime}, 0.32$, and 0.44 , respectively. The spectrometer used was a filter bank with $250 \mathrm{kHz}$ resolution, with two independent sections of 128 channels each. The filter bank was put in parallel configuration, in which each of the two sections received independent signals with a different polarization. The parallel configuration was chosen so that ultimately the two polarizations could be averaged to produce spectra with a better signal-to-noise ratio $(\mathrm{S} / \mathrm{N})$. At a frequency of $230 \mathrm{GHz}$, the resultant velocity resolution for this setup is $0.65 \mathrm{~km} \mathrm{~s}^{-1}$.

The on-the-fly (OTF) mapping technique was used to observe an $11^{\prime} \times 37.5$ area along a position angle of $49^{\circ}$ surrounding the axis of the HH 300 flow's redshifted lobe. The OTF technique allowed the extended area to be mapped in a more efficient way than the conventional point-by-point mapping. The telescope in OTF mode moved across the source at a constant speed of $30^{\prime \prime} \mathrm{s}^{-1}$, while data were taken at a rate of 10 samples $\mathrm{s}^{-1}$. In order to map the desired area more efficiently, the total area mapped consists of overlapping regions of different sizes: one large-sized map of $8^{\prime} \times 33^{\prime}$, one medium-sized map of $8^{\prime} \times 20^{\prime}$, and two small-sized maps of $3^{\prime} \times 6^{\prime}$ and $8^{\prime} \times 5^{\prime}$. All the regions were scanned along the direction perpendicular to the HH flow's axis, which has a P.A. $=49^{\circ}$. The separation, in the direction perpendicular to the scanning direction, between subsequent rows was $6^{\prime \prime}$. The telescope was pointed to an OFF position, located at R.A. $4^{\mathrm{h}} 21^{\mathrm{m}} 40^{\mathrm{s}}$, decl. $24^{\circ} 05^{\prime} 00^{\prime \prime}(\mathrm{B} 1950)$, after every other row, where it would observe the OFF position for $10 \mathrm{~s}$ and then vane calibrate for $5 \mathrm{~s}$. The off position was later observed to have emission in the velocity range between 2.4 and $6.3 \mathrm{~km} \mathrm{~s}^{-1}$, with 0.1 $\mathrm{K} \lesssim T_{A}^{*} \lesssim 0.5 \mathrm{~K}$, and no emission greater than $0.1 \mathrm{~K}$ at other velocities. The emission from the red lobe of the $\mathrm{HH}$ 300 outflow is at velocities greater than $6.7 \mathrm{~km} \mathrm{~s}^{-1}$. Therefore, the small amount of emission at velocities between 2.4 and $6.3 \mathrm{~km} \mathrm{~s}^{-1}$ at the OFF position does not affect our outflow data (see Fig. 2). The different regions were 


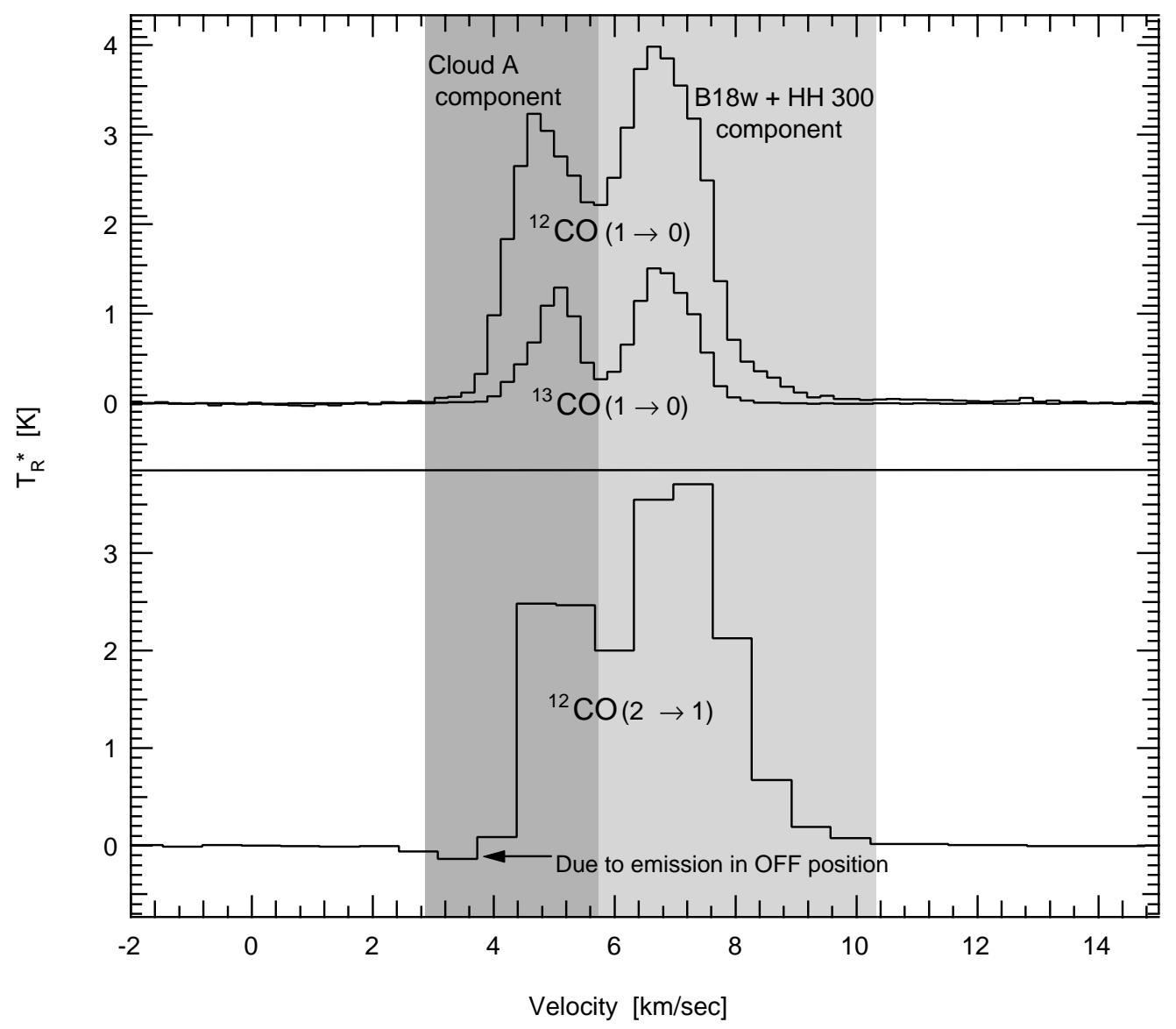

FIG. 2.-(Top) Average ${ }^{12} \mathrm{CO}(1-0)$ and ${ }^{13} \mathrm{CO}(1-0)$ spectra of the area mapped in ${ }^{12} \mathrm{CO}(2-1)$, which includes most of the HH 300 outflow red lobe and some cloud regions unaffected by the outflow. (Bottom) Average ${ }^{12} \mathrm{CO}(2-1)$ spectrum over the same area as the spectra above [the area covered by the ${ }^{12} \mathrm{CO}$ (2-1) map].

observed several times to improve the $\mathrm{S} / \mathrm{N}$ in the spectra. The system temperature was measured to be in the range between 400 and $900 \mathrm{~K}$. In order to keep a constant noise level through the whole map, the regions that were mapped with a higher system temperature were observed more than the regions with lower system temperature.

The raw OTF data were reduced using the various Astronomical Image Processing System OTF tasks. A firstorder baseline was fitted and subtracted to each spectrum, and the two polarizations were averaged. The different mapped regions were combined and averaged. The map was then convolved onto a grid with 14" pixels. The resultant rms noise in each $0.65 \mathrm{~km} \mathrm{~s}^{-1}$ channel was $0.12 \mathrm{~K}$ for most (nonborder) spectra. The intensity scale of the spectral data presented in this paper is in units of $T_{A}^{*}$ (Kutner \& Ulich 1981) unless it is stated otherwise.

$$
\text { 2.2. }{ }^{12} \mathrm{CO}(1-0),{ }^{13} \mathrm{CO}(1-0) \text {, and } \mathrm{C}^{18} \mathrm{O}(1-0) \text { Data }
$$

In order to study the effect of the HH 300 outflow on a larger scale and to attain a better estimate of the outflow mass, we observed the ${ }^{12} \mathrm{CO}(1-0)$ and ${ }^{13} \mathrm{CO}(1-0)$ lines in a region $17^{\prime} .5 \times 59^{\prime}$ along a position angle of $49^{\circ}$ surrounding the HH 300 outflow source (IRAS 04239+2436). The data were obtained using the SEQUOIA 16-element focal plane array receiver of the Five College Radio Astronomy Observatory (FCRAO) $14 \mathrm{~m}$ telescope. The observations were done over the course of three different observing rounds, which took place in 1999 April and December and 2000
February. Both lines were observed in frequency-switched mode, and the back end used was the Focal Plane Array Autocorrelator Spectrometer, with a channel spacing of 78 $\mathrm{kHz}\left(0.21 \mathrm{~km} \mathrm{~s}^{-1}\right)$ for ${ }^{12} \mathrm{CO}(1-0)$, at a frequency of 115 $\mathrm{GHz}$, and $20 \mathrm{kHz}\left(0.05 \mathrm{~km} \mathrm{~s}^{-1}\right)$ for ${ }^{13} \mathrm{CO}(1-0)$, at a frequency of $110 \mathrm{GHz}$. The telescope half-power beamwidths for the ${ }^{12} \mathrm{CO}(1-0)$ and ${ }^{13} \mathrm{CO}(1-0)$ lines are $45^{\prime \prime}$ and $47 "$, and the main beam efficiencies are 0.45 and 0.54 , respectively. The ${ }^{12} \mathrm{CO}(1-0)$ spectra were taken with a spacing of $44^{\prime \prime}$ (beam sampled) and with an integration time of $150 \mathrm{~s}$ for each position. The ${ }^{13} \mathrm{CO}(1-0)$ spectra were taken with a spacing of 22" (Nyquist sampled) and with an integration time of $100 \mathrm{~s}$ for each position. The system temperature of our observations ranged between 700 and $1000 \mathrm{~K}$ for ${ }^{12} \mathrm{CO}$ $(1-0)$ and between 300 and $500 \mathrm{~K}$ for ${ }^{13} \mathrm{CO}(1-0)$.

Observations of the $\mathrm{C}^{18} \mathrm{O}(1-0)$ emission were also made using the same telescope and back end configuration as the ${ }^{13} \mathrm{CO}(1-0)$ observations. We made three Nyquist-sampled FCRAO-SEQUOIA footprints. The 5.5 × 5.5 footprints were centered at offsets $(0,0),\left(-3{ }^{\prime} 1,-3^{\prime} \cdot 1\right)$, and $\left(-6^{\prime} .9\right.$, $\left.-9{ }^{\prime} 4\right)$ with respect to the outflow source position at $(\alpha, \delta)_{1950}=\left(4^{\mathrm{h}} 23^{\mathrm{m}} 54^{\mathrm{s}} .5,24^{\circ} 36^{\prime} 54^{\prime \prime}\right)$. The telescope halfpower beamwidth and main beam efficiency for $\mathrm{C}^{18} \mathrm{O}(1-0)$ at a frequency of $109 \mathrm{GHz}$ are $47^{\prime \prime}$ and 0.54 , respectively. Each position was observed for $200-300 \mathrm{~s}$, with a system temperature ranging from 170 to $350 \mathrm{~K}$.

The ${ }^{12} \mathrm{CO}(1-0)$ and ${ }^{13} \mathrm{CO}(1-0)$ data were reduced with the CLASS and MIRIAD packages. The original spectra 
from both lines were smoothed and resampled to a channel spacing of $0.22 \mathrm{~km} \mathrm{~s}^{-1}$. The spectra were spatially linearly interpolated, and a data cube of 11 ".5 pixels was produced for each line. The data cube was then smoothed by convolving it with a Gaussian with an FWHM of 46" using the task "smooth" in MIRIAD. The resultant rms noise of the spectra in the ${ }^{12} \mathrm{CO}(1-0)$ and ${ }^{13} \mathrm{CO}(1-0)$ smoothed maps was 0.17 and $0.07 \mathrm{~K}$ for each $0.22 \mathrm{~km} \mathrm{~s}^{-1}$ channel, respectively. Interpolating the ${ }^{12} \mathrm{CO}(1-0)$ and the ${ }^{13} \mathrm{CO}(1-0)$ data to this common position-velocity grid allowed us to use them in concert when calculating the mass estimates presented in $\S 3.3 .2$.

\section{RESULTS}

Figure 2 shows spectra of ${ }^{12} \mathrm{CO}(2-1),{ }^{12} \mathrm{CO}(1-0)$, and ${ }^{13} \mathrm{CO}(1-0)$ averaged over the area mapped in ${ }^{12} \mathrm{CO}(2-1)$. The most notable property of the spectra in Figure 2 is that they are composed of two main (Gaussian-like) components. One component is centered at a velocity close to $6.7 \mathrm{~km} \mathrm{~s}^{-1}$, and the other component is centered at a velocity close to $5 \mathrm{~km} \mathrm{~s}^{-1}$. The $v=6.7 \mathrm{~km} \mathrm{~s}^{-1}$ component is produced by the molecular emission of the HH 300 outflow host cloud (B18w), while the other component, at $v=5 \mathrm{~km}$ $\mathrm{s}^{-1}$, is produced by another cloud in Taurus (hereafter cloud A) along the same line of sight. Unlike many other average outflow spectra (e.g., HH 111, Cernicharo \& Reipurth 1996; NGC 2264G, Lada \& Fich 1996; RNO 43, Bence et al. 1996), the red wing of the ${ }^{12} \mathrm{CO}(2-1)$ and ${ }^{12} \mathrm{CO}$ (1-0) average spectra is not very prominent. The wing extends only from about 8 to about $10 \mathrm{~km} \mathrm{~s}^{-1}$. In addition to the small red wing, the line core component of the $\mathrm{B} 18 \mathrm{w}$ cloud seems to have a slight asymmetry, with more emission toward red velocities. This suggests that the $\mathrm{HH} 300$ outflow is composed mainly of gas with slow radial velocities and the gas emission of its slowest velocities might be hidden under the ambient cloud emission.

The cloud A component is visible not only in the area surrounding the red lobe of $\mathrm{HH} 300$ but also through most of the area that we mapped in ${ }^{12} \mathrm{CO}(1-0)$ and ${ }^{13} \mathrm{CO}(1-0)$. This area includes the region where the blue lobe of HH 300 should be, assuming that it lies northeast of IRAS $04239+2436$, opposite to the red lobe at the same position angle. There is evidence in the optical (RBD) and nearinfrared (Reipurth et al. 2000) for blueshifted outflowing material only very close to the source. But, even if the $\mathrm{HH}$ 300 flow's blue lobe were interacting with the surrounding gas (close to and/or far from the source), we would not be able to study it. The "contamination" from cloud A on the blue side of the B18w component is such that we would not be able to observe the high-velocity blue wings that presumably would have been present in the ${ }^{12} \mathrm{CO}$ spectra. Therefore, we concentrate our study on the interaction between the redshifted lobe of the $\mathrm{HH} 300$ flow and its surroundings.

\section{1. ${ }^{13} \mathrm{CO}(1-0)$ Maps}

Figure 3 shows five ${ }^{13} \mathrm{CO}(1-0)$ velocity maps, integrated over five different velocity ranges. The first map is integrated over the velocity in which the emission from cloud A is present, $3.79 \mathrm{~km} \mathrm{~s}^{-1}<v<5.77 \mathrm{~km} \mathrm{~s}^{-1}$. The other three maps are made over velocity ranges in which there is emission from the B18w cloud (and HH 300 outflow). It is evident that the velocity map in Figure $3 a$ shows a ${ }^{13} \mathrm{CO}$ (1-0) structure different from the other velocity maps. In addition, the position angle of the short and long axes of the
$3.79 \mathrm{~km} \mathrm{~s}^{-1}<v<5.77 \mathrm{~km} \mathrm{~s}^{-1}$ structure differs from that of the B18w cloud by about $30^{\circ}$. This reconfirms our assumption that the emission from velocities $3.8-5.8 \mathrm{~km} \mathrm{~s}^{-1}$ does not come from the $\mathrm{B} 18 \mathrm{w}$ cloud but from another cloud, cloud A, on the same line of sight.

The integrated velocity map over the range $5.77 \mathrm{~km}$ $\mathrm{s}^{-1}<v<7.09 \mathrm{~km} \mathrm{~s}^{-1}$ is shown in Figure $3 b$. The velocity range of Figure $3 b$ does not cover the whole range of velocities in which there is emission from the B18w cloud component $\left(5.77 \mathrm{~km} \mathrm{~s}^{-1}<v<8.63 \mathrm{~km} \mathrm{~s}^{-1}\right)$ in Figure 2. But, even though the map in Figure $3 b$ only shows emission from 5.77 to $7.09 \mathrm{~km} \mathrm{~s}^{-1}$, it shows the general ${ }^{13} \mathrm{CO}(1-0)$ structure of the B18w cloud. Comparing Figure $3 b$ with an integrated map that covers the whole velocity range of emission from the B18w component $\left(5.77 \mathrm{~km} \mathrm{~s}^{-1}<v<8.63 \mathrm{~km}\right.$ $\mathrm{s}^{-1}$ ) shows that both maps have essentially the same structure. And, comparing Figure $3 b$ with a map integrated over just $6.2-7.1 \mathrm{~km} \mathrm{~s}^{-1}$ also shows that they have similar structure. In addition, the shape of these ${ }^{13} \mathrm{CO}(1-0)$ maps is very similar to the shape of the B18w dark cloud as seen in the optical image of RBD (see Fig. 6). Therefore, the emission from the $\mathrm{B} 18 \mathrm{w}$ cloud is predominantly from gas at velocities between 6.2 and $7.1 \mathrm{~km} \mathrm{~s}^{-1}$, the peak emission range for the spectra in Figure 2.

The integrated intensity map over $7.09 \mathrm{~km}$ $\mathrm{s}^{-1}<v<7.53 \mathrm{~km} \mathrm{~s}^{-1}$ has a very peculiar structure (Fig. $3 c$ ). The long and short axes as well as the position angle of this structure are similar to those of the $5.77 \mathrm{~km}$ $\mathrm{s}^{-1}<v<7.09 \mathrm{~km} \mathrm{~s}^{-1}$ (Fig. 3b) structure. Also, the $7.09 \mathrm{~km}$ $\mathrm{s}^{-1}<v<7.53 \mathrm{~km} \mathrm{~s}^{-1}$ structure bears some resemblance to the ${ }^{12} \mathrm{CO}(1-0)$ outflow in the velocity range of $7.53 \mathrm{~km}$ $\mathrm{s}^{-1}<v<7.97 \mathrm{~km} \mathrm{~s}^{-1}$ (see Fig. 5a). The local maximum in ${ }^{13} \mathrm{CO}(1-0)$ at R.A. $4^{\mathrm{h}} 22^{\mathrm{m}} 50^{\mathrm{s}}$, decl. $24^{\circ} 21^{\prime} 30^{\prime \prime}(\mathrm{B} 1950)$ is at the tip of the most prominent bow shock-like clump (clump R3; see $\S 3.2$ and Figs. 4, 5, and 6) in the ${ }^{12} \mathrm{CO}$ outflow. Although it is tempting to conclude that most of the emission at these velocities comes from the "slow moving" outflowing gas, the velocity of this structure (about $7.75 \mathrm{~km}$ $\left.\mathrm{s}^{-1}\right)$ is still very close to the line center velocity $\left(V_{\mathrm{LSR}}=6.67\right.$ $\mathrm{km} \mathrm{s}^{-1}$ ). Thus, we expect some contribution to the emission at $7.09 \mathrm{~km} \mathrm{~s}^{-1}<v<7.53 \mathrm{~km} \mathrm{~s}^{-1}$ to come from the turbulent gas in $\mathrm{B} 18 \mathrm{w}$ unaffected by the outflow. We are convinced that ${ }^{13} \mathrm{CO}(1-0)$ emission from both slow moving outflowing gas and ambient cloud gas are present in the integrated intensity map in the velocity range of $7.09 \mathrm{~km}$ $\mathrm{s}^{-1}<v<7.53 \mathrm{~km} \mathrm{~s}^{-1}$ (see $\S 3.3 .3$ for a quantitative discussion).

Figure $3 d$ shows the integrated intensity for the velocity range $7.53 \mathrm{~km} \mathrm{~s}^{-1}<v<7.75 \mathrm{~km} \mathrm{~s}^{-1}$. There is no doubt that the ${ }^{13} \mathrm{CO}(1-0)$ emission in this velocity range, southwest of IRAS $04239+2436$, is due to gas that has been put in motion by the outflow. The ${ }^{13} \mathrm{CO}(1-0)$ emission coincides in position with the medium-velocity structure seen in the ${ }^{12} \mathrm{CO}$ outflow velocity maps (see Figs. 4 and 5). The emission detected northeast of the outflow source at these redshifted velocities, with respect to the ambient cloud velocity $\left(V_{\mathrm{LSR}}=6.67 \mathrm{~km} \mathrm{~s}^{-1}\right)$, is not associated with the $\mathrm{HH}$ 300 flow. Instead, this emission comes from an elongated structure perpendicular to the HH 300 flow's axis, associated with the B18 molecular cloud, as can be seen in the large-scale, 2.7-resolution, ${ }^{13} \mathrm{CO}(1-0)$ velocity maps of Taurus by Mizuno et al. (1995). The integrated intensity map over the range $7.75 \mathrm{~km} \mathrm{~s}^{-1}<v<8.63 \mathrm{~km} \mathrm{~s}^{-1}$ (see Fig. $3 e$ ) shows the highest (red) velocities of the HH 300 outflow 

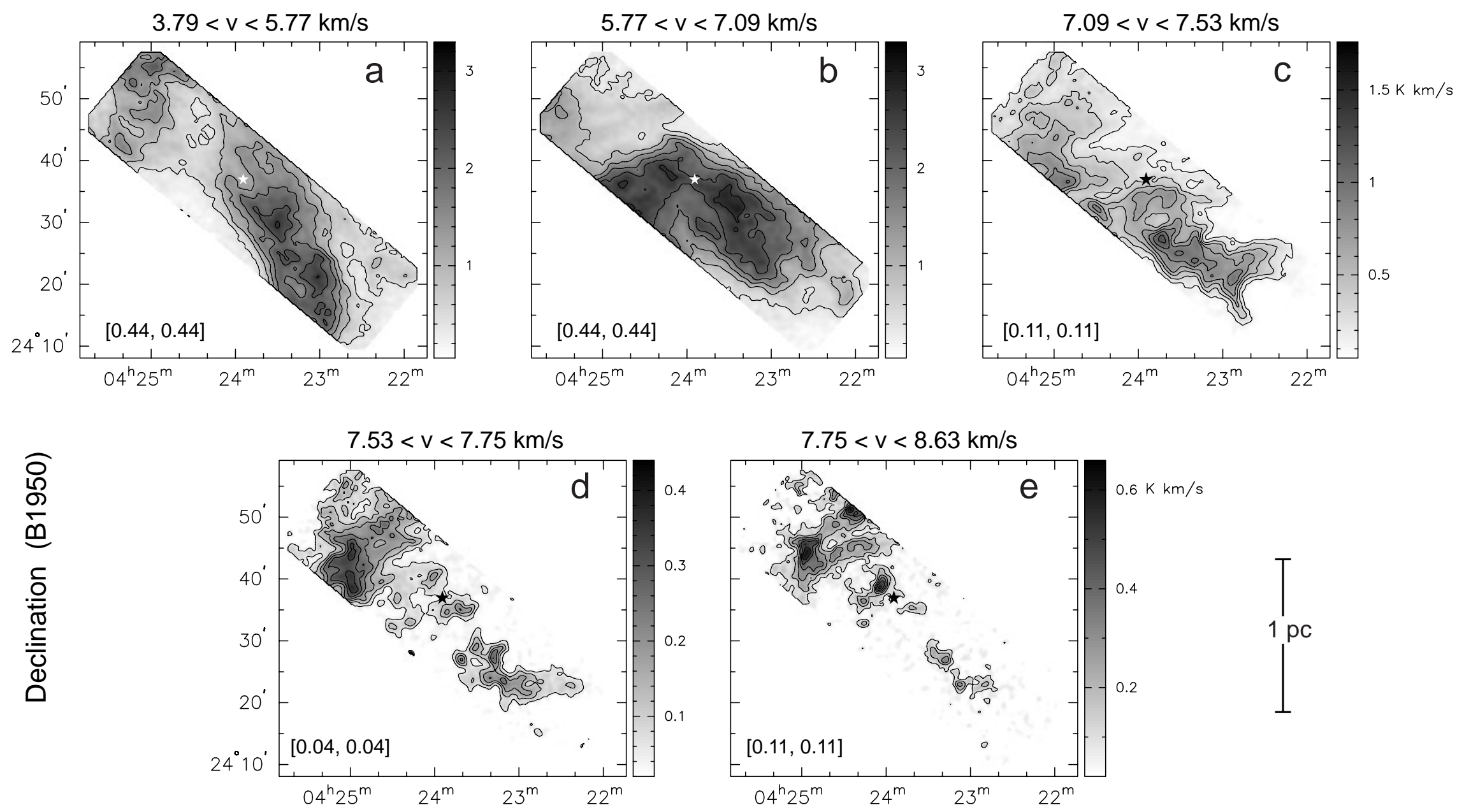

\section{Right Ascension (B1950)}

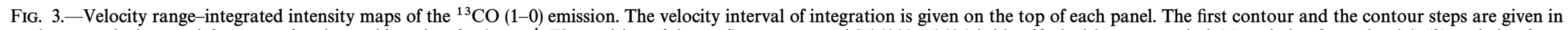

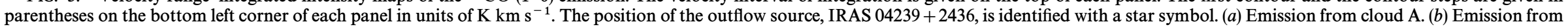

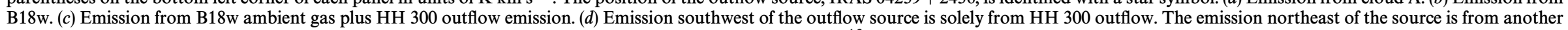
cloud on the same line of sight. (e) Emission southwest of outflow source is from the highest velocity outflowing ${ }^{13} \mathrm{CO}(1-0)$ gas. 


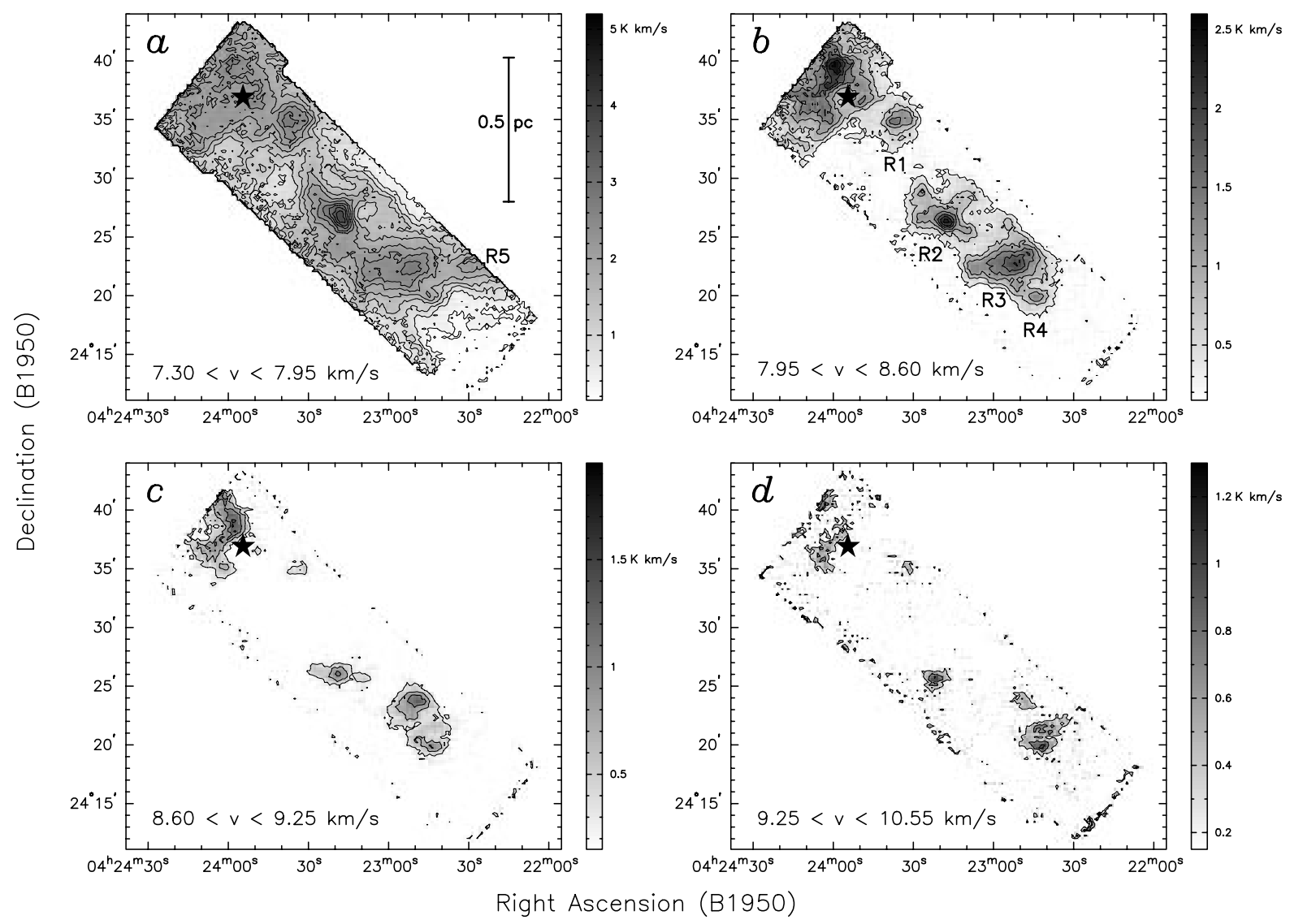

FIG. 4.-Velocity range-integrated intensity maps of the ${ }^{12} \mathrm{CO}(2-1)$ emission from the redshifted lobe of the HH 300 molecular outflow. The velocity interval of integration is given on the bottom of each panel. The first contour and the contour steps are $0.325 \mathrm{~K} \mathrm{~km} \mathrm{~s}^{-1}$ for all maps. The position of the outflow source, IRAS $04239+2436$, is identified with a star symbol. The five outflow clumps R1, R2, R3, R4, and R5 are labeled.

in ${ }^{13} \mathrm{CO}(1-0)$ emission. The knotty features of this highvelocity ${ }^{13} \mathrm{CO}(1-0)$ coincide with the position of the highvelocity features in the ${ }^{12} \mathrm{CO}$ outflow (see Figs. 4 and 5 ). These knotty features are well defined in space and velocity.

\section{2. ${ }^{12} \mathrm{CO}$ Maps}

In Figures 4 and 5 we show ${ }^{12} \mathrm{CO}(2-1)$ and ${ }^{12} \mathrm{CO}(1-0)$ velocity maps of the $\mathrm{HH} 300$ molecular outflow. The ${ }^{12} \mathrm{CO}$
(1-0) data set covers more area and has a better spectral resolution than the ${ }^{12} \mathrm{CO}(2-1)$ data. On the other hand, the ${ }^{12} \mathrm{CO}(2-1)$ data has a better spatial resolution and sensitivity than the ${ }^{12} \mathrm{CO}(1-0)$ data. Regardless of the differences in spectral and spatial resolution, area, and sensitivity, the ${ }^{12} \mathrm{CO}(2-1)$ and ${ }^{12} \mathrm{CO}(1-0)$ maps look very similar for similar velocity ranges (see Figs. 4 and 5). The most notable characteristic of this outflow is its very clumpy structure.

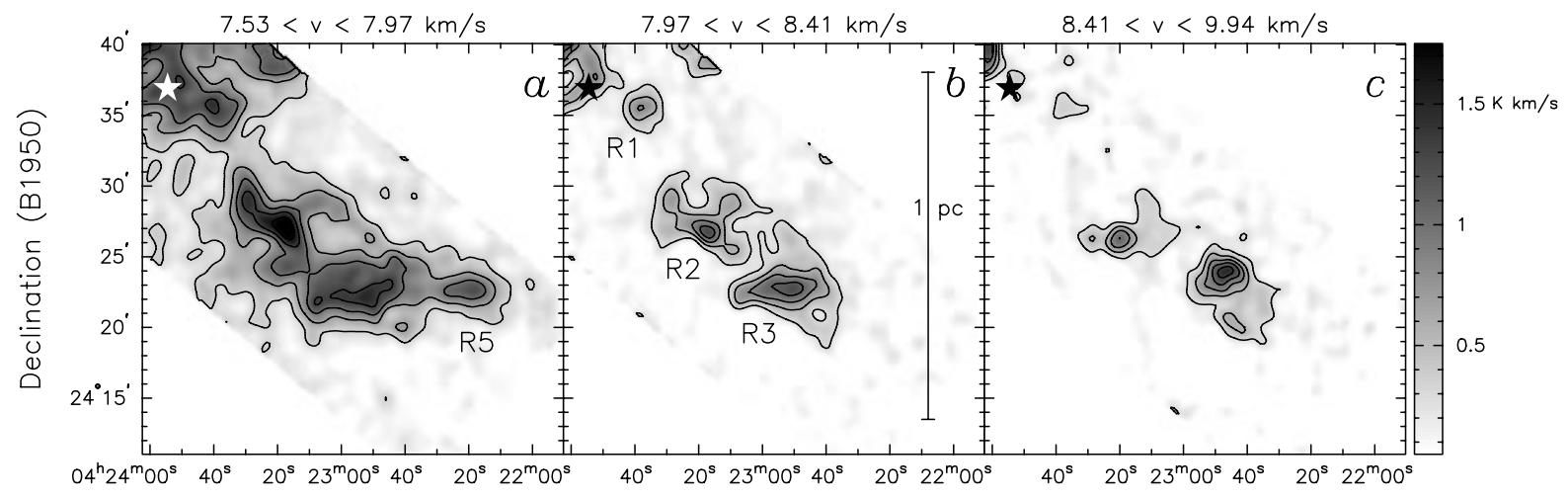

Right Ascension (B1950)

FIG. 5.- Velocity range-integrated intensity maps of the ${ }^{12} \mathrm{CO}(1-0)$ emission from the redshifted lobe of the HH 300 molecular outflow. The velocity interval of integration is given on the top of each panel. The first contour and the contour steps are $0.22 \mathrm{~K} \mathrm{~km} \mathrm{~s}^{-1}$ for all maps. The position of the outflow source, IRAS $04239+2436$, is identified with a star symbol. Four of the five outflow clumps (R1, R2, R3, and R5) are labeled. 


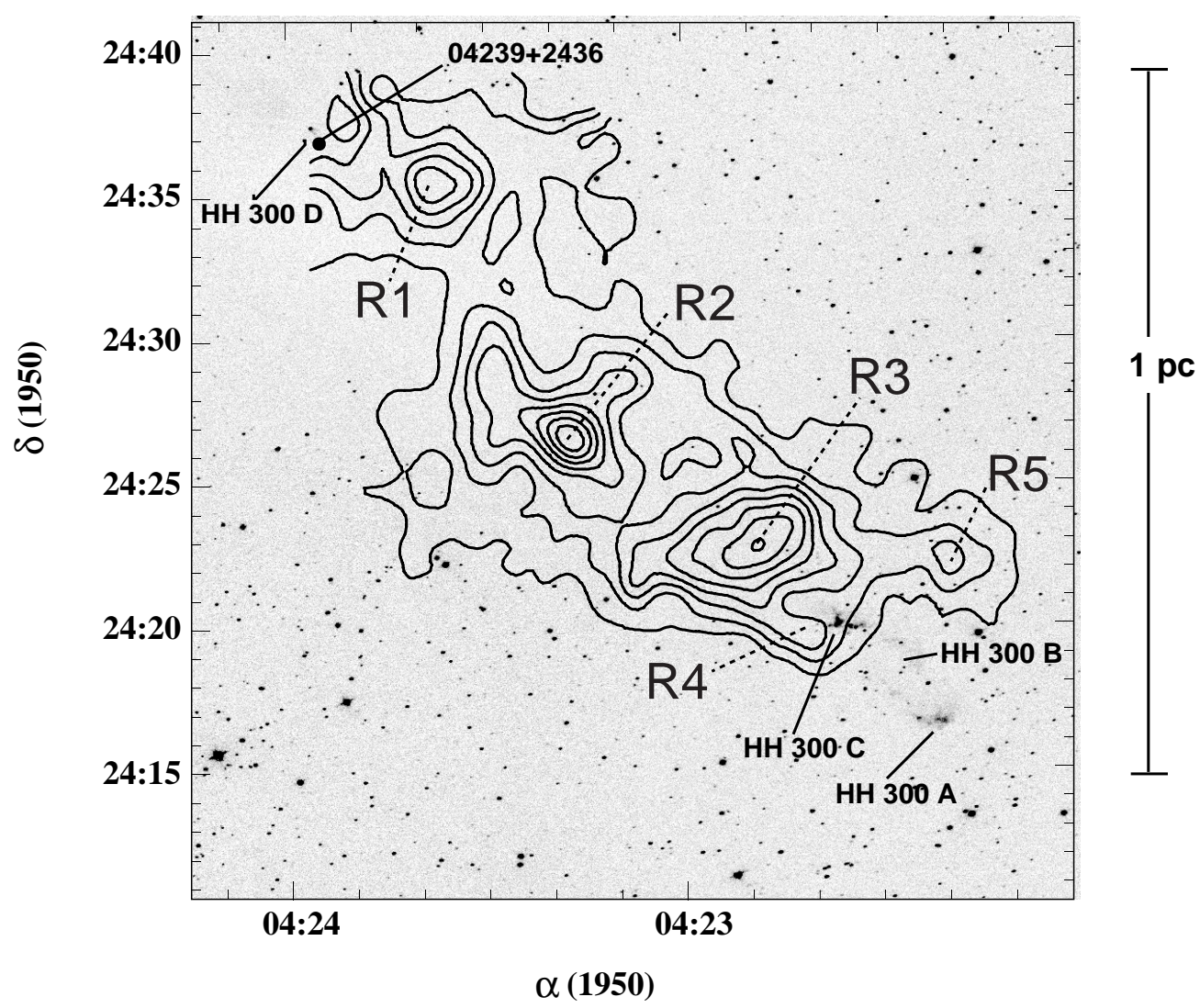

Fig. 6.-Integrated intensity contour map of the ${ }^{12} \mathrm{CO}(1-0)$ emission over the range $7.53 \mathrm{~km} \mathrm{~s}^{-1}<v<9.94 \mathrm{~km} \mathrm{~s}^{-1}$, superimposed on a wide-field $\mathrm{H} \alpha+\left[\mathrm{S}\right.$ II] (optical) CCD image from RBD. The starting contour of the integrated intensity map is $0.55 \mathrm{~K} \mathrm{~km} \mathrm{~s}^{-1}$, and the contour steps are $0.275 \mathrm{~K} \mathrm{~km} \mathrm{~s} \mathrm{~s}^{-1}$. The A, B, C, and D HH knot groups from the HH 300 flow are labeled. We also label the molecular outflow clumps R1, R2, R3, R4, and R5. The location of the deeply embedded outflow source, IRAS $04239+2436$, is shown. The B18w cloud is seen in the optical image as a region with no background stars (due to extinction).

We identify five molecular clumps in the redshifted lobe of the HH 300 outflow. In Figure 6 and Table 1, we show how five named clumps (R1-R5) are identified.

We believe that all five clumps mentioned above are associated with the HH 300 outflow and each clump represents a different ejection event. Clumps R1 and R2 have "blobby" structures like the high-velocity $\mathrm{CO}$ bullets found in HH 111 by Cernicharo \& Reipurth (1996). Clump R3 has a shape reminiscent of a bow shock, with wings pointing in the direction of the outflow source (see Figs. $4 b$ and $5 b$ ). There is stronger ${ }^{12} \mathrm{CO}$ emission in the south wing than in the north wing of the bow structure. This asymmetry in the

TABLE 1

DeteCtability of Clumps in HH 300 Redshifted Lobe

\begin{tabular}{|c|c|c|c|c|c|}
\hline \multirow[b]{2}{*}{ Clump } & \multicolumn{3}{|c|}{ INTEGRATED INTENSITY MAPS ${ }^{a}$} & \multicolumn{2}{|c|}{ Position-Velocity DiagRams ${ }^{b}$} \\
\hline & ${ }^{13} \mathrm{CO}(1-0)$ & ${ }^{12} \mathrm{CO}(1-0)$ & ${ }^{12} \mathrm{CO}(2-1)$ & ${ }^{12} \mathrm{CO}(1-0)$ & ${ }^{12} \mathrm{CO}(2-1)$ \\
\hline $\mathrm{R} 1 \ldots .$. & Yes & Yes & Yes & Yes & Yes \\
\hline $\mathrm{R} 2 \ldots \ldots$ & Yes & Yes & Yes & Yes & Yes \\
\hline $\mathrm{R} 3 \ldots \ldots$ & $\mathrm{Yes}^{\mathrm{c}}$ & Yes & Yes & Yes & Yes \\
\hline $\mathrm{R} 4 \ldots \ldots$ & No & $\ldots^{d}$ & Yes & $\ldots{ }^{\mathrm{e}}$ & Yes \\
\hline $\mathrm{R} 5 \ldots \ldots$ & No & Yes & Yes $^{f}$ & No & Yes \\
\hline
\end{tabular}

${ }^{a}$ Here we note if the clump structure can be seen in any of the integrated intensity velocity maps of the different molecular lines (Figs. 3, 4, and 5).

${ }^{b}$ Here we note if the clump shows a velocity increase in the position-velocity (PV) diagrams (Figs. 9 and 10).

${ }^{c}$ The $\mathrm{R} 3$ clump is seen as two clumps in ${ }^{13} \mathrm{CO}(1-0)$.

d There is ${ }^{12} \mathrm{CO}(1-0)$ emission at the position of clump R4, but the ${ }^{12} \mathrm{CO}(1-0)$ maps do not show the same clearly defined clump structure as in the ${ }^{12} \mathrm{CO}(2-1)$ maps.

${ }^{e}$ There is not a clear peak in the velocity at the position of clump R4 in the ${ }^{12} \mathrm{CO}(1-0) \mathrm{PV}$ diagram, though there is high-velocity gas (see Fig. 10).

${ }^{f}$ Clump R5 can only be seen partly in the ${ }^{12} \mathrm{CO}(2-1)$ data because of the limited extent of the ${ }^{12} \mathrm{CO}(2-1)$ map (see Fig. 4a). 
bow wings of R3 is most probably due to the underlying distribution of molecular gas in the cloud, since there is more gas emission south of clump R3 than to its north. Recent high spatial resolution observations of outflows (e.g., Yu et al. 1999; Lee et al. 2000) have found that bow-shaped $\mathrm{CO}$ structures are common. The $\mathrm{CO}$ bow structures presumably are created by the interaction between a bow shock from an underlying jet and the ambient gas. Clump R4 is barely distinguishable as a separate clump from R3, but it is still clear that R4 exhibits a local maximum of $\mathrm{CO}$ emission and that it diverges from the bow structure of R3 (see Fig. 4b). We are confident that R4 is associated with the HH 300 outflow not only due to its relatively (redshifted) high velocity but also because it coincides in position with the optical knot HH 300C (see Fig. 6). The molecular clump R5 is the clump with the slowest velocities and can only be seen in the velocity maps with velocities close to the cloud velocity. It has an elongated droplike structure, as if it were a bow shock, with only one wing. The same effect that produces asymmetric bow wings in $\mathrm{R} 3$ could be producing the structure of R5, but in a more dramatic way. In the case of R5, there is practically no molecular emission north of the clump, but there is some emission south of it. It is very probable that we do not see a north bow wing in R5, since there is essentially no gas to the north of the clump to produce $\mathrm{a}^{12} \mathrm{CO}$ wing. Table 2 shows the distance from the source and the position angle, as well as other physical properties of each of the molecular clumps.

\subsection{Outflow Physical Properties}

In order to study the effects of a molecular outflow on its environment, it is essential to calculate its mass, momentum, and energy in the most precise way possible. There are several potential pitfalls that, if not avoided or compensated for, can reduce the precision of the calculation of an

TABLE 2

\begin{tabular}{lcccc}
\multicolumn{5}{c}{ Physical PropertiEs OF Clumps IN HH 300} \\
REDSHIFT LOBE \\
\hline \hline Clump & $\begin{array}{c}d^{\mathrm{a}} \\
(\mathrm{pc})\end{array}$ & $\begin{array}{c}\text { Angle }^{\mathrm{b}} \\
(\mathrm{deg})\end{array}$ & $\begin{array}{c}\mathrm{Age}^{\mathrm{c}} \\
(\mathrm{yr})\end{array}$ & $\begin{array}{c}\mathrm{Mass}^{\mathrm{d}} \\
\left(M_{\odot}\right)\end{array}$ \\
\hline $\mathrm{R} 1 \ldots \ldots$ & 0.16 & 250 & 800 & 0.07 \\
$\mathrm{R} 2 \ldots \ldots$ & 0.53 & 221 & 2500 & 0.21 \\
$\mathrm{R} 3 \ldots \ldots$ & 0.81 & 228 & 4000 & $0.23^{\mathrm{e}}$ \\
$\mathrm{R} 4 \ldots \ldots$ & 0.98 & 225 & 4800 & $\ldots \mathrm{e}^{\mathrm{e}}$ \\
$\mathrm{R} 5 \ldots \ldots$ & 1.03 & 238 & 5000 & $0.02^{\mathrm{f}}$
\end{tabular}

a Distance from the outflow source to the maximum emission point on clump.

${ }^{b}$ Position angle (east of north) of line intersecting outflow source and maximum emission point on clump.

${ }^{c}$ Dynamical age of the clump assuming mass ejection from source, responsible for the clump, has a tangential velocity of $200 \mathrm{~km} \mathrm{~s}^{-1}$.

${ }^{\mathrm{d}}$ Mass of clump for $v_{\text {out }} \geq 0.97 \mathrm{~km} \mathrm{~s}^{-1}$, uncorrected for cloud emission. Emission at $v_{\text {out }} \geq 0.97$ $\mathrm{km} \mathrm{s}^{-1}$ is dominated by outflow clump emission; cloud emission dominates at slower velocity channels.

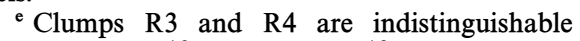
(unresolved) in ${ }^{12} \mathrm{CO}(1-0)$ and ${ }^{13} \mathrm{CO}(1-0)$ lines; the total mass of both is given in the R3 mass entry.

${ }^{\mathrm{f}}$ Unlike the other clumps, clump R5 is seen better at lower velocities, $v_{\text {out }} \sim 0.75 \mathrm{~km} \mathrm{~s}^{-1}$. Mass of clump R5 for $v_{\text {out }} \geq 0.75 \mathrm{~km} \mathrm{~s}^{-1}$ is $0.11 M_{\odot}$. outflow's physical properties. Major dangers include (1) not accounting for the inclination of the flow's axis to the plane of the sky, (2) assuming that the molecular spectral line being used to obtain the physical properties is optically thin when in reality it is optically thick, and (3) not counting slow moving outflow gas that is hidden by the ambient cloud molecular emission as part of the flow. With our data, we have found a way to tackle the uncertainties produced by (2) and (3). On the other hand, (1) remains a problem.

\subsubsection{Inclination}

The most accurate way of obtaining $i$ for a molecular outflow is to observe the tangential $\left(V_{t}\right)$ and radial $\left(V_{r}\right)$ velocities of an $\mathrm{HH}$ object associated with the molecular outflow being studied. Assuming that the $\mathrm{HH}$ object travels through space along a straight line and that the molecular gas from the molecular outflow associated with the $\mathrm{HH}$ object will move in the same direction, then $i=$ $\arctan \left(V_{t} / V_{r}\right)$. Unfortunately, no proper motion study of the HH 300 optical knots has been done; thus, we do not know the transverse velocities of the $\mathrm{HH} 300$ knots. Other methods of estimating the value of $i$ for molecular outflows use a simple geometrical and velocity field model for the flow, combined with the observed data of the outflow (see Liseau \& Sandell 1986; Cabrit, Goldsmith, \& Snell 1988). Using these models results in $i$-values with large uncertainties, but at least they give a rough estimate to the value of $i$ when otherwise there would be none. The clumpy structure of the HH 300 molecular outflow (very different from the molecular outflow cone geometry assumed by the models listed above) and the fact that it is impossible to detect any blueshifted $\mathrm{CO}$ emission from the $\mathrm{HH} 300$ outflow due to contamination from cloud A make it impossible to use the simple geometric models to estimate $i$ for $\mathrm{HH}$ 300. In addition, if the HH 300 outflow is precessing and episodic (see $\S 4.3$ ), then each mass eruption could have been ejected at a different angle with respect to the plane of the sky, so that using a single value of $i$ for HH 300 would be an incorrect assumption.

The HH 300 flow most likely lies very close to the plane of the sky. Most of the giant $\mathrm{HH}$ flows for which the inclination angle is known have been found to have small values of $i$. By definition, giant $\mathrm{HH}$ flows are flows that are observed to extend more than $1 \mathrm{pc}$ in length on the sky. The smaller the angle between the flow's axis and the plane of the sky, the longer the projected (on the plane of the sky) size of a flow will be for a given flow length. Thus, it is not surprising that there is a bias toward small values of $i$ in giant $\mathrm{HH}$ flows. The small maximum outflow radial velocities ( 3-4 $\mathrm{km} \mathrm{s}^{-1}$ ) that the HH 300 molecular outflow exhibits, as well as the morphology of the near-infrared nebula surrounding the outflow source (as observed by Reipurth et al. 2000), further confirm that HH 300 lies very close to the plane of the sky. We believe that the (average) axis of the HH 300 flow must have an inclination angle with respect to the plane of the sky between $5^{\circ}$ and $15^{\circ}$. In this paper, all outflow velocities quoted for HH 300 are not corrected by inclination angle, unless otherwise noted.

\subsubsection{Opacity Correction}

Using an optically thick line to study an outflow will cause an underestimation of the mass and kinetic energy in the flow. One should worry not only about the line core opacity but also about the dependence of the line opacity 
on velocity. Using the outflow mass estimation methods of Bally et al. 1999 (hereafter BRLB) and Yu et al. 1999 (hereafter YBB) with our ${ }^{13} \mathrm{CO}(1-0)$ and ${ }^{12} \mathrm{CO}(1-0)$ data in $\mathrm{HH} 300$, we believe we have obtained a fairly accurate estimate of the outflow mass.

We do not strictly follow BRLB's or YBB's method but use a combination of the two, with our own modifications. First, we take an average of all the ${ }^{13} \mathrm{CO}(1-0)$ and ${ }^{12} \mathrm{CO}$ (1-0) spectra in the area southwest of IRAS $04239+2436$, where the outflow lies. Then, we plot the ratio of the lines as a function of velocity (see Fig. 7a). The ${ }^{12} \mathrm{CO}(1-0)$ line is optically thick over all velocities where there is detectable ${ }^{13} \mathrm{CO}(1-0)$, as the line ratio is always less than 60 . It is clear that the line ratio (and thus the opacity) is not constant over velocity and that it has a parabolic shape. Assuming a constant opacity for the ${ }^{12} \mathrm{CO}(1-0)$ line, as in most studies that claim to correct for the ${ }^{12} \mathrm{CO}(1-0)$ opacity using the ${ }^{13} \mathrm{CO}$ (1-0) line, is not an accurate assumption. It is imperative to take the velocity dependence of the opacity into consideration.

Using the average spectra of the two molecular lines, we obtain the value of the main beam temperature $\left(T_{\mathrm{mb}}\right)$ for each $0.22 \mathrm{~km} \mathrm{~s}^{-1}$-wide channel and calculate the ratio of main beam temperatures. We then make a second-order polynomial fit to the ratio of ${ }^{12} \mathrm{CO}(1-0)$ to ${ }^{13} \mathrm{CO}(1-0)$ intensity, $R_{12 / 13}$, as a function of velocity. The fit is constrained to have a minimum at the velocity of the ambient cloud [equal to the velocity where the average ${ }^{13} \mathrm{CO}(1-0)$ spectrum peaks], $V_{\mathrm{LSR}}=6.67 \mathrm{~km} \mathrm{~s}^{-1}$. We exclude the five velocity channels closest to the ambient velocity from the fit, as they are the velocities at which the ${ }^{13} \mathrm{CO}$ emission is slightly optically thick (see below) and the ${ }^{12} \mathrm{CO}$ emission is
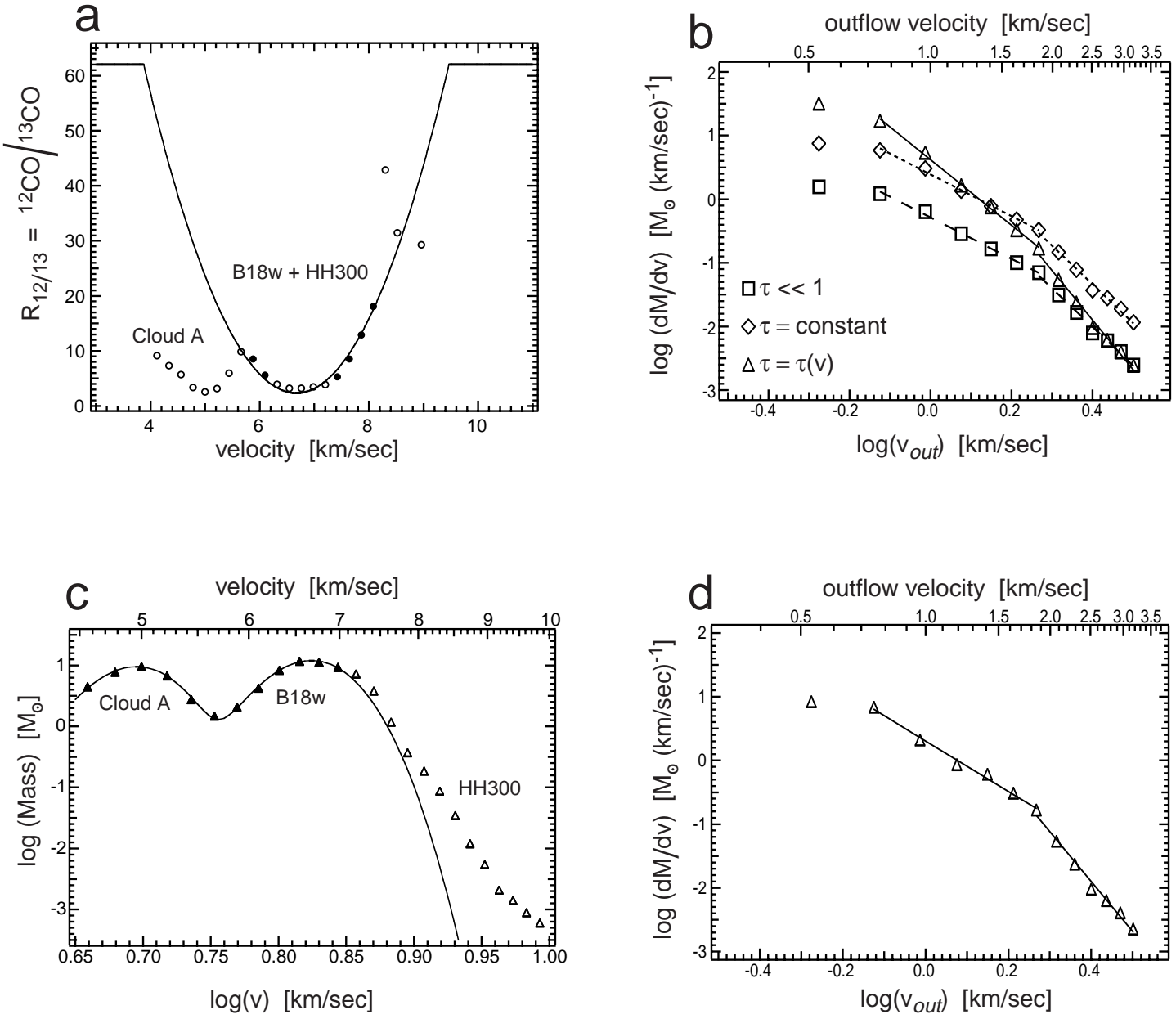

Fig. 7.- (a) Main beam temperature (or intensity) ratio of ${ }^{12} \mathrm{CO}(1-0)$ to ${ }^{13} \mathrm{CO}(1-0)$, also denoted in the text as $R_{12 / 13}$, as a function of observed velocity (v). The filled circles are the points used for the second-order polynomial fit to $R_{12 / 13}$. The solid line is the resulting fit. (b) Mass spectrum, or mass in a $0.22 \mathrm{~km}$ $\mathrm{s}^{-1}$-wide channel as a function of outflow velocity $\left(v_{\text {out }}=v-V_{\mathrm{LSR}}\right)$, for the red lobe of the HH 300 molecular outflow. The squares denote the mass obtained assuming that ${ }^{12} \mathrm{CO}(1-0)$ is optically thin (mass $\propto \int T_{\mathrm{mb}} d v$ ). The diamonds represent the mass obtained assuming that the ${ }^{12} \mathrm{CO}(1-0)$ line opacity is constant $\left(R_{12 / 13}=13\right)$. The triangles denote the mass assuming that $R_{12 / 13}$, and thus the opacity of the ${ }^{12} \mathrm{CO}(1-0)$ line, varies as a function of velocity, as shown in $(a)$. For each of these three different mass estimates, we show two power-law fits, one for low outflow velocities between 0.75 and $1.85 \mathrm{~km} \mathrm{~s}^{-1}$ and another for high outflow velocities between 1.85 and $3.17 \mathrm{~km} \mathrm{~s}^{-1}$. The points at $v_{\text {out }}=0.53 \mathrm{~km} \mathrm{~s}^{-1}$ are real but are excluded from the fits, as they lie too close to the ambient line core. Velocities are not corrected for outflow inclination. The long- and short-dashed lines are power-law fits to the optically thin mass estimate and the constant opacity mass estimate, respectively. The solid lines show power-law fits to the velocity-dependent opacity-corrected mass spectrum. The power-law exponents from the fits are as follows: $\tau \ll 1:-3.3 \pm 0.1$ (low velocity), $-6.1 \pm 0.2$ (high velocity) $\tau=$ constant: $-3.3 \pm 0.1$ (low velocity), $-6.1 \pm 0.2$ (high velocity); $\tau(v):-5.2 \pm 0.1$ (low velocity), $-7.8 \pm 0.4$ (high velocity). (c) Mass in a $0.22 \mathrm{~km} \mathrm{~s}^{-1}$-wide channel as a function of observed velocity. Solid line is a two-Gaussian fit to the filled triangle symbols (points that are not contaminated by emission from the HH 300 outflow). (d) Mass spectrum corrected for ambient cloud emission. The mass is corrected for ambient cloud emission by subtracting the modeled ambient cloud mass (given by the Gaussian fit in $[c]$ ) from the velocity-dependent opacity-corrected mass (shown in $[b]$ in triangle symbols). See discussion in text (§ 3.3.3). Similar to (b), the resultant power-law fits are shown by solid lines. The slopes of the fits are $-4.0 \pm 0.2$ and $-7.8 \pm 0.4$. 
extremely optically thick. Also, we use only the points that lie between the velocities 5.8 and $8.2 \mathrm{~km} \mathrm{~s}^{-1}$, in order to exclude cloud $\mathrm{A}$ and low-S/N velocities. We use the $R_{12 / 13}(v)$ parabolic fit to extrapolate $R_{12 / 13}$ to the highvelocity wings of the outflow, where the ${ }^{13} \mathrm{CO}(1-0)$ line is too weak to be reliably detected.

We estimate the mass in the following way: If the ${ }^{13} \mathrm{CO}$ (1-0) emission at a velocity $v_{i}$ at a certain position $\left(x_{i}, y_{i}\right)$ is greater than or equal to twice the rms noise of the spectrum at that position, we use the main beam temperature value at the given velocity $\left[T_{\mathrm{mb}}^{13}\left(x_{i}, y_{i}, v_{i}\right)\right]$. If the ${ }^{13} \mathrm{CO}(1-0)$ emission is less than twice the rms noise, then we use the ${ }^{12} \mathrm{CO}(1-0)$ spectrum at the given position $\left(x_{i}, y_{i}\right)$ and velocity $v_{i}$ to estimate $T_{\mathrm{mb}}^{13}\left(x_{i}, y_{i}, v_{i}\right)=T_{\mathrm{mb}}^{12}\left(x_{i}, y_{i}, v_{i}\right)\left[R_{12 / 13}\left(v_{i}\right)\right]^{-1}$. Similar to what BRLB and YBB do, the function $R_{12 / 13}(v)$ is truncated at a value of 62 , the assumed isotopic ratio (Langer \& Penzias 1993). If both the ${ }^{13} \mathrm{CO}(1-0)$ and the ${ }^{12} \mathrm{CO}(1-0)$ emissions at $\left(x_{i}, y_{i}, v_{i}\right)$ are less than twice the rms noise, then essentially there is no signal from which we can estimate a value of $T_{\mathrm{mb}}^{13}\left(x_{i}, y_{i}, v_{i}\right)$.

Once we obtain the value of $T_{\mathrm{mb}}^{13}\left(x_{i}, y_{i}, v_{i}\right)$, we can get the value of the opacity, assuming that the ${ }^{13} \mathrm{CO}(1-0)$ line is optically thin. Using our $\mathrm{C}^{18} \mathrm{O}$ data, we are able to estimate the opacity of the ${ }^{13} \mathrm{CO}(1-0)$ emission at several positions. The $\mathrm{C}^{18} \mathrm{O}$ observations were made where strong ${ }^{13} \mathrm{CO}$ (1-0) emission was found, i.e., regions where the ${ }^{13} \mathrm{CO}(1-0)$ emission might be optically thick. From the ${ }^{13} \mathrm{CO}$ to $\mathrm{C}^{18} \mathrm{O}$ ratio, we estimate the opacity of the ${ }^{13} \mathrm{CO}(1-0)$ line (as a function of velocity) at the limited positions for which we have $\mathrm{C}^{18} \mathrm{O}$ data. The ${ }^{13} \mathrm{CO}-$ to- $^{18} \mathrm{O}$ ratios indicate that even in regions with strong ${ }^{13} \mathrm{CO}$ emission, the opacity of the ${ }^{13} \mathrm{CO}(1-0)$ line at line core velocities $(6.2 \mathrm{~km}$ $\mathrm{s}^{-1}<v<7.0 \mathrm{~km} \mathrm{~s}^{-1}$ ) does not exceed a value of 3 , and for other velocities the ${ }^{13} \mathrm{CO}$ emission is optically thin. Since we are mainly interested in the outflow mass, our assumption that ${ }^{13} \mathrm{CO}(1-0)$ is optically thin is an excellent assumption.

At each position and at each velocity for which we have an estimate of $T_{\mathrm{mb}}^{13}\left(x_{i}, y_{i}, v_{i}\right)$, we calculate the ${ }^{13} \mathrm{CO}(1-0)$ opacity, $\tau_{13}(x, y, v)$, using

$\tau_{13}(x, y, v)=-\ln \left\{1-\frac{T_{\mathrm{mb}}^{13}(x, y, v)}{T_{0} /\left[\exp \left(T_{0} / T_{\mathrm{ex}}\right)-1\right]-0.87}\right\}$

from Rohlfs \& Wilson (1996). Here $T_{0}=h v / k$, which for ${ }^{13} \mathrm{CO}(1-0)$ is 5.29 . The value of $\tau_{13}$, from equation (1), agrees with the value of the ${ }^{13} \mathrm{CO}$ opacity obtained using the ${ }^{13} \mathrm{CO}-$ to- $\mathrm{C}^{18} \mathrm{O}$ line ratio in the limited number of positions where we measured the $\mathrm{C}^{18} \mathrm{O}$ emission. The excitation temperature, $T_{\mathrm{ex}}$, is obtained by assuming that the ${ }^{12} \mathrm{CO}$ (1-0) line core is optically thick. We take an average spectrum of the ${ }^{12} \mathrm{CO}(1-0)$ data over all of the area southwest of the source for which we have ${ }^{12} \mathrm{CO}(1-0)$ data and measure the peak temperature (corrected for main beam efficiency) to be $T_{\text {peak }}=9.6 \mathrm{~K}$. Solving the radiative transfer equation for the excitation temperature, assuming that the line is optically thick, we obtain this expression,

$$
T_{\text {ex }}=\frac{5.53}{\ln \left[1+5.53 /\left(T_{\text {peak }}+0.82\right)\right]},
$$

from Estalella \& Anglada (1997). The resultant $T_{\mathrm{ex}}$ is $13 \mathrm{~K}$, the same value that Mizuno et al. (1995) quote for the whole Taurus molecular cloud complex. Then, at each position and velocity the column density, $N_{13}(x, y, v)$, can be calcu- lated with the following equation from Bourke et al. (1997):

$$
N_{13}(x, y, v)=2.42 \times 10^{14}\left(T_{\mathrm{ex}}+0.88\right) \frac{\tau_{13}(x, y, v) d v}{1-\exp \left(-T_{0} / T_{\mathrm{ex}}\right)} .
$$

The outflow (molecular hydrogen) mass at each position pixel and each velocity channel is given by

$$
M(x, y, v)=m_{\mathrm{H}_{2}} N_{\mathrm{H}_{2}}(x, y, v) A,
$$

where $m_{\mathrm{H}_{2}}$ (which is equal to 2.72 times the mass of a hydrogen atom) is the mean molecular weight (taking into consideration the abundance of helium and other molecules), $N_{\mathrm{H}_{2}}$ is the molecular hydrogen column density that is obtained using the relation $N_{\mathrm{H}_{2}}=7 \times 10^{5} N_{13}$ (Frerking, Langer, \& Wilson 1982), and $A$ is the physical area of the pixel at the distance of the source. We then sum over the area of the outflow $\left[M(v)=\sum_{\text {area }} M(x, y, v)\right]$ to obtain the outflow mass as a function of velocity and then sum over velocity $\left[M_{\text {total }}=\sum_{v} M(v)\right]$ to obtain the total outflow mass. The uncertainty in the mass estimates comes mainly from the uncertainty in the $N_{\mathrm{H}_{2}}$ to $N_{13}$ ratio; thus, the uncertainty in the mass estimates is about a factor of 2 (Frerking et al. 1982).

Using this method we can calculate the mass per $0.22 \mathrm{~km}$ $\mathrm{s}^{-1}$-wide velocity channel $[d M(v) / d v]$ in the HH 300 flow. In Figure $7 b$ we plot our results for outflow velocities $\left(v_{\text {out }}\right)$ greater than $0.5 \mathrm{~km} \mathrm{~s}^{-1}$, where $v_{\text {out }}$ is the observed velocity (v) minus the cloud velocity $\left(V_{\mathrm{LSR}}=6.67\right)$. Figure $7 b$ shows that the observed mass (per $0.22 \mathrm{~km} \mathrm{~s}^{-1}$-wide velocity channel), corrected only for the velocity-dependent opacity of the ${ }^{12} \mathrm{CO}(1-0)$ line, has a power-law dependence on velocity for $v_{\text {out }}$ between 0.75 and $1.85 \mathrm{~km} \mathrm{~s}^{-1}$. For outflow velocities higher than $1.85 \mathrm{~km} \mathrm{~s}^{-1}$, the mass has an even steeper power-law dependence. A power-law fit to each of these two trends yields a slope of $-5.2 \pm 0.1$ for the lowvelocity points and a slope of $-7.8 \pm 0.4$ for the highvelocity points.

\subsubsection{Correction for Ambient Cloud Emission}

We suspect that some velocity channels have emission from both the low-velocity outflowing gas and the ambient cloud. Thus, in order to obtain an accurate estimate of the outflow mass, it is essential to correct for the "extra mass" at low velocities due to ambient cloud contamination. In order to make this correction, we use our calculations of the mass per velocity channel (§3.3.2) for $4.5 \mathrm{~km} \mathrm{~s}^{-1}<v<9.8$ $\mathrm{km} \mathrm{s}^{-1}$. Using the overall (cloud A + B18w + HH 300) mass spectrum in Figure 7c, we fit a two-Gaussian function to the points that define the clouds (cloud A and B18w) and that we are sure are not contaminated by the $\mathrm{HH} 300$ outflow (see Fig. 7c). The Gaussians, which seem a good fit to the relevant ambient emission, give us a value of $V_{\mathrm{LSR}}=$ $6.67 \mathrm{~km} \mathrm{~s}^{-1}$ as the center velocity of the B18w component, which is the value we use as the velocity of the cloud throughout the paper, and a center velocity for cloud A of $4.95 \mathrm{~km} \mathrm{~s}^{-1}$. The outflow emission can clearly be seen in Figure $7 c$ as a deviation from the Gaussian shape at redshifted velocities. To obtain the corrected outflow mass $\left[M_{\text {cor }}(v)\right]$, we subtract the value of the fit at the velocity of interest $\left[M_{\mathrm{fit}}(v)\right]$ from the observed mass $\left[M_{\mathrm{obs}}(v)\right]$. Using the simple relation $M_{\text {cor }}(v)=M_{\text {obs }}(v)-M_{\mathrm{fit}}(v)$, we obtain an outflow mass that is presumably free from the cloud emission. We display the mass-velocity relation using the cor- 
rected mass in Figure $7 d$. It can be seen that the lowvelocity power-law slope of the mass spectrum (Fig. 7d) becomes shallower, with a slope of $-4.0 \pm 0.2$, after the correction. From here on all the outflow mass (and momentum) values given are corrected for ambient cloud emission, unless otherwise noted.

We note that this method would result in an underestimation of mass in the lowest outflow velocity channels if the emission were extremely optically thick at these velocities or if the points used for the cloud fit were heavily affected by outflow emission. As discussed above, neither of these two scenarios is our case, so we are confident that our underestimation of low-velocity outflow mass is minimal. The mass and the power-law slope at high outflow velocities $\left(v_{\text {out }}>\right.$ $1.85 \mathrm{~km} \mathrm{~s}^{-1}$ ) are unchanged after the ambient cloud correction, since there is no contamination from the ambient cloud at those velocities.

\subsubsection{Effects of Inclination, Opacity, and Ambient Contamination}

In estimating an outflow's momentum and energy, corrections for inclination can have profound effects, since we can only observe a radial velocity. On the other hand, the slope of the mass spectrum (see Fig. 7b) is not affected by a flow's inclination. Correcting the velocity for projection effects will just rescale the velocity axis of the mass spectrum plot, but the slope of the power-law mass spectrum will remain the same, assuming that all parts of the flow have roughly the same inclination angle, $i$.

The effects that velocity-dependent opacity correction have on the outflow's mass and mass spectrum can be seen clearly in Figure $7 b$. In this figure we plot the mass spectrum of the red lobe of $\mathrm{HH} 300$, using three different assumptions. The square symbols denote the mass spectrum obtained assuming that the ${ }^{12} \mathrm{CO}(1-0)$ line is optically thin, so that mass is proportional to $\int T_{\mathrm{mb}} d v$. The diamond symbols represent the mass spectrum obtained by assuming that the ${ }^{12} \mathrm{CO}(1-0)$ line opacity, and thus $R_{12 / 13}=$ $I\left({ }^{12} \mathrm{CO}\right) / I\left({ }^{13} \mathrm{CO}\right)$, is constant. We use the area- and velocityaveraged value of $R_{12 / 13}$ over the velocity range between 7.3 and $8.5 \mathrm{~km} \mathrm{~s}^{-1}$ (or outflow velocities between 0.75 and $1.85 \mathrm{~km} \mathrm{~s}^{-1}$ ) as an average opacity, $\tau_{\text {avg. }}$. The mass is then calculated using equations (1), (3), and (4) and assuming that $T_{\mathrm{mb}}^{13}=T_{\mathrm{mb}}^{12} / \tau_{\mathrm{avg}}$, with $\tau_{\mathrm{avg}}=13$. The triangle symbols show the mass spectrum obtained using the technique described in $\$ 3.3 .2$.

From Figure $7 b$ it is clear that assuming ${ }^{12} \mathrm{CO}(1-0)$ is optically thin greatly underestimates the mass (by a factor of 10) compared to the opacity-corrected estimates. The constant opacity correction gives a more realistic estimate of the total mass. The shape and slopes of the mass spectrum using the constant opacity correction and the mass spectrum obtained using the optically thin assumption are basically the same. Assuming that the opacity of the ${ }^{12} \mathrm{CO}$ $(1-0)$ is constant implies that the high-velocity ${ }^{12} \mathrm{CO}(1-0)$ emission is optically thick. Since it is clear that the ${ }^{12} \mathrm{CO}$ (1-0) line is optically thick at outflow velocities close to the cloud $V_{\text {LSR }}$ and it is optically thin at high velocities, a more precise approach is to assume that the ${ }^{12} \mathrm{CO}(1-0)$ line opacity depends on velocity. The velocity-dependent opacity correction results in low-velocity mass estimates that are close to the constant opacity assumption, and at high velocities the mass estimates converge to the optically thin assumption estimates. This steepens the mass spectrum in both the low- and high-velocity regimes.
Comparing Figures $7 b$ and $7 d$, we see that the correction for ambient cloud emission makes the mass spectrum shallower. This is because there is more contamination from the ambient cloud at lower velocities than at higher velocities. Not correcting for such contamination results in an overestimation of the mass at lower velocities. Thus, correcting for a velocity-dependent opacity and correcting for ambient cloud emission have opposite effects on the mass spectrum slopes. Most previous outflow studies do neither of the two corrections, so it could be that their estimates of the mass spectrum slope are reasonable by chance.

\section{DISCUSSION}

\subsection{Mass and Energetics}

The total mass of the red lobe of the HH 300 outflow, using the method described in $\S \S 3.3 .2$ and 3.3.3, for $v_{\text {out }} \geq$ $0.75 \mathrm{~km} \mathrm{~s}^{-1}$, is $2.4 M_{\odot}$. As can be seen in Figure $7 d$, the mass-velocity relation at velocities between 0.75 and 1.85 $\mathrm{km} \mathrm{s}^{-1}$ follows a power-law trend, $[d M(v) / d v] \propto v^{-\gamma}$. A second power-law, with a steeper slope, is present for velocities greater than $1.85 \mathrm{~km} \mathrm{~s}^{-1}$. This broken power-law trend is typical of many outflows (see $\S 4.2$ ). If we take "outflow mass" to include only mass at velocities that follow the broken power-law trend, then we find $2.4 M_{\odot}$ in the outflow. Notice the lone point at $v_{\text {out }}=0.53 \mathrm{~km} \mathrm{~s}^{-1}$, which lies below the extrapolation of the power-law trend in Figure $7 d$. If we add the mass at this very slow outflow velocity (corrected by ambient emission; see $\S 3.3 .3$ ), we then find the total mass of the red lobe of $\mathrm{HH} 300$ outflow to be $4.3 M_{\odot}$ for $v_{\text {out }} \geq 0.53 \mathrm{~km} \mathrm{~s}^{-1}$. The mass of the ambient cloud gas that surrounds the red lobe of HH 300 (only the B18w gas southwest of IRAS $04239+2436)$ is $57 M_{\odot}$; thus, the mass in the outflow, including the slowest velocity material that we can reliably call "outflow," is $7 \%$ of the mass of its surrounding cloud.

The total (line of sight) momentum in the red lobe of the HH 300 molecular outflow for $v_{\text {out }} \geq 0.75 \mathrm{~km} \mathrm{~s}^{-1}$ is $2.2 M_{\odot}$ $\mathrm{km} \mathrm{s}^{-1}$ and for $v_{\text {out }} \geq 0.53 \mathrm{~km} \mathrm{~s}^{-1}$ is $3.2 M_{\odot} \mathrm{km} \mathrm{s}^{-1}$. The amount of momentum that has been deposited in the cloud by HH 300 presumably should be substantially more, as we have only considered the line-of-sight velocity component. If we assume that $i$ is between $5^{\circ}$ and $15^{\circ}$, then the outflow momentum would be between 12 and $37 M_{\odot} \mathrm{km} \mathrm{s}^{-1}$ for $v_{\text {out }} \geq 0.53 \mathrm{~km} \mathrm{~s}^{-1}$. Thus, our ignorance of $i$ brings huge uncertainties to the value of the outflow momentum. The kinetic energy of the outflow is even more uncertain, as it depends on $(\sin i)^{-2}$. The kinetic energy of the outflow for $v_{\text {out }} \geq 0.75 \mathrm{~km} \mathrm{~s}^{-1}$ is $2.1(\sin i)^{-2} \times 10^{43}$ ergs and for $v_{\text {out }} \geq$ $0.53 \mathrm{~km} \mathrm{~s}^{-1}$ is $2.6(\sin i)^{-2} \times 10^{43}$ ergs. So, for example, if $i=10^{\circ}$, the kinetic energy is about $8 \times 10^{44}$ ergs.

Independent of the real (angle corrected) momentum, we can still map line-of-sight outflow momentum. In Figure 8 we show a contour plot of the total momentum for $v_{\text {out }} \geq$ $0.97 \mathrm{~km} \mathrm{~s}^{-1}$ (not corrected for inclination angle). We are sure that there is very little or no emission from the ambient cloud at these velocities. The momentum plot shows distinctive peaks along the outflow axis. These peaks coincide with the different clumps discussed in $\S 3.2$. The peaks are surrounded by a $\sim 0.3 \mathrm{pc}-$ wide and $\sim 1 \mathrm{pc}-$ long lower level momentum distribution.

We assume that the dimension along the line of sight of both $\mathrm{B} 18 \mathrm{w}$ and the $\mathrm{HH} 300$ outflow are similar to their respective short axes. Using the maps in Figure 8, we esti- 


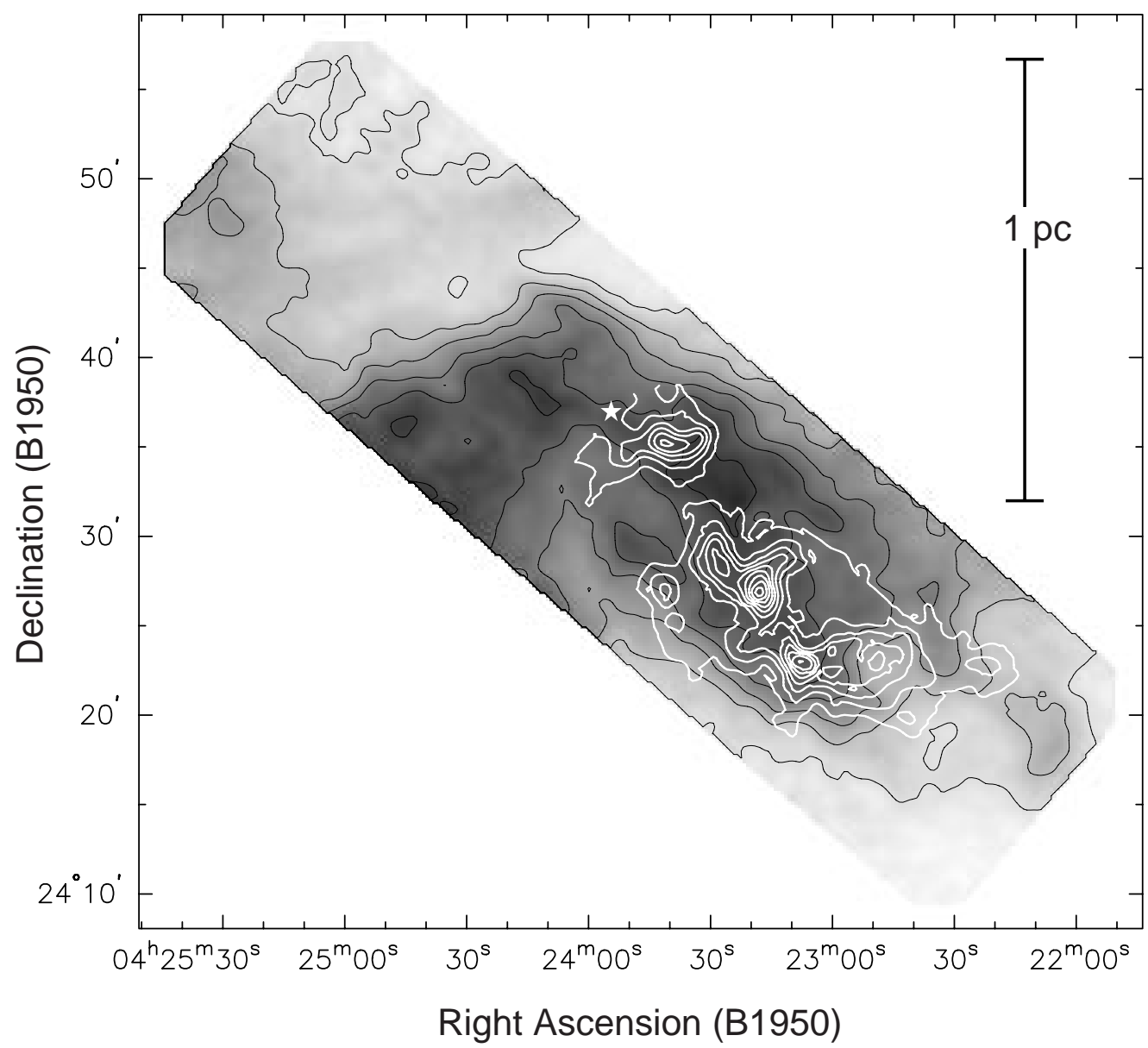

FIG. 8.-Contour map of outflow total momentum, not corrected for inclination angle, for outflow velocities greater than $0.97 \mathrm{~km} \mathrm{~s}{ }^{-1}$. The momentum contour map is superimposed on a B18w gray-scale map of ${ }^{13} \mathrm{CO}(1-0)$ integrated intensity for $5.8 \mathrm{~km} \mathrm{~s}^{-1}<v<7.1 \mathrm{~km} \mathrm{~s}{ }^{-1}$, as shown in Fig. $3 b$. The momentum was obtained using the velocity-dependent opacity correction technique described in the text. The starting contour and the contour steps on the momentum map are both $1.5 \times 10^{-4} M_{\odot} \mathrm{km} \mathrm{s}^{-1}$.

mate their volumes to be $1.7 \times 0.7 \times 0.7 \sim 0.83 \mathrm{pc}^{3}$ and $1.0 \times 0.3 \times 0.3 \sim 0.09 \mathrm{pc}^{3}$, respectively. Hence, we may conclude that a notable fraction $\left(0.09 \mathrm{pc}^{3} / 0.83 \mathrm{pc}^{3} \sim 11 \%\right.$ of the volume) of the B18w filamentary dark cloud is being injected with momentum from the red lobe of the HH 300 outflow. The mass, momentum, and kinetic energy estimates of the HH 300 outflow are listed in Table 3.

\subsection{Mass-Velocity Relation}

An interesting aspect of the HH 300 outflow is the steep power-law slopes that we find in the mass-velocity relation $\left(\gamma=4.0\right.$ for $v_{\text {out }} 0.75-1.85 \mathrm{~km} \mathrm{~s}^{-1}$ and $\gamma=7.8$ for $v_{\text {out }} \geq 1.85$ $\mathrm{km} \mathrm{s}^{-1}$ ). All molecular outflows exhibit a power-law massvelocity relation in which there is more outflowing mass at low velocities than at higher velocities; that is, $[d M(v) /$ $d v] \propto v^{-\gamma}$, where $\gamma>0$. Most outflows show a break in the power-law relation and a steeper power-law dependence at high velocities. Recently, Richer et al. (2000) compiled ex- isting data on 22 molecular outflows with central source luminosity ranging from $0.58 L_{\odot}$ to $3 \times 10^{5} L_{\odot}$. The lowvelocity $\left(v<10 \mathrm{~km} \mathrm{~s}^{-1}\right)$ mass spectra $\gamma \mathrm{s}$ for the 22 sources range from about 0.25 to 2.5 , with most of the values concentrating between 1.25 and 2 . For high velocities, $\gamma$ ranges from 2.5 to 7.5, with most of the values between 3 and 4 .

Numerical and theoretical studies have also shown that the mass spectrum has a power-law dependence. The models of Zhang \& Zheng (1997) and Smith, Suttner, \& Yorke (1997), in which a molecular outflow is produced by the entrainment of the ambient gas by a bow shock, find a $\gamma$ of about 1.8 and steepening of the mass-velocity relation at high velocities. Downes \& Ray (1999), with their numerical bow shock models, find that the slope of the powerlaw mass spectrum ranges between $\gamma=1.58$ and 3.75 (depending on the parameters of the simulation). Both Smith et al. (1997) and Downes \& Ray (1999) find that $\gamma$ increases with time. On the other hand, the analytical study

TABLE 3

Mass, Momentum, and Kinetic EnERgy of the Redshifted Lobe of HH 300

\begin{tabular}{ccc}
\hline \hline Parameter & $v_{\text {out }} \geq 0.75 \mathrm{~km} \mathrm{~s}^{-1}$ & $v_{\text {out }} \geq 0.53 \mathrm{~km} \mathrm{~s}^{-1}$ \\
\hline Mass $\left(M_{\odot}\right) \ldots \ldots \ldots \ldots \ldots \ldots \ldots . \ldots \ldots \ldots \ldots$ & 2.4 & 4.3 \\
Momentum $\left(M_{\odot} \mathrm{km} \mathrm{s}^{-1}\right) \ldots \ldots \ldots$. & $2.2(\sin i)^{-1}$ & $3.2(\sin i)^{-1}$ \\
Kinetic energy $\left(\times 10^{43} \mathrm{ergs}\right) \ldots \ldots$ & $2.1(\sin i)^{-2}$ & $2.6(\sin i)^{-2}$ \\
\hline
\end{tabular}


by Matzner $\&$ McKee (1999) finds that $\gamma=2$ for all times, independent of the time history of the driving wind and for a wide variety of ambient density distributions. The study of Matzner \& McKee (1999) differs from the other ones listed above in that the outflow is not specifically created by the entrainment of the ambient gas by a bow shock but rather by a wide-angle wind that sweeps the ambient gas into a momentum-conserving shell.

The majority of observations and models in the literature to date seem to indicate that $\gamma \sim 2$. Our observations indicate that the HH 300 outflow has a much steeper $(\gamma \sim 4)$ mass spectrum than most outflows (from low- and intermediate-mass stars), both observed and modeled. On the other hand, our results are similar to the outflows analyzed by BRLB, YBB, and Yu et al. 2000 (hereafter YBSBB). In Table 4 we show the average value of $\gamma$ for the outflows where mass estimates have included a correction for velocity-dependent ${ }^{12} \mathrm{CO}$-line opacity. From Table 4 we can see that after this correction most of the outflows have power-law slopes larger than 2. A small difference between the results in this paper and those of BRLB and YBB is that we find a steepening of the mass spectrum power-law for high velocities in HH 300, whereas BRLB and YBB state that the outflows in their studies do not show such steepening. Examining the mass spectrum plots of BRLB and $\mathrm{YBB}$, it is clear that some of them do not show a break in the power law. Yet, others of their mass spectrum plots could be fitted with two distinct power laws, where the high-velocities would have a steeper $\gamma$ than the lowvelocities.

There is no question that the ${ }^{12} \mathrm{CO}$ line is optically thick in the $\mathrm{HH} 300$ flow and that its opacity depends on velocity, as the ${ }^{12} \mathrm{CO}(1-0) /{ }^{13} \mathrm{CO}(1-0)$ plot (Fig. 7a) demonstrates. The same is true for the Circinus (BRLB), the B5-IRS 1 (YBB), and the MMS 9 (YBSBB) outflows. It is safe to say that the ${ }^{12} \mathrm{CO}$ line of most, if not all, outflows is optically thick at low velocities and that its opacity is velocity dependent. Thus, if low-velocity ${ }^{12} \mathrm{CO}$ emission is used to estimate the outflow mass and no correction is made for a velocity-dependent opacity, the resultant mass spectrum power-law slope will be underestimated. This can be shown using our data. If we assume that ${ }^{12} \mathrm{CO}(1-0)$ is optically thin or that it has a constant (velocity independent) opacity, we obtain $\gamma \sim 3.2$ for the HH 300 outflow mass spectrum (uncorrected for ambient emission), while the velocitydependent opacity-corrected value is $\sim 5.2$ (see Fig. $7 b$ ).
But, the fact that the HH 300, B5-IRS 1, and Circinus outflows all have large values of $\gamma$ does not necessarily mean that $\gamma$ would dramatically increase for all other outflows if they were to be corrected for velocity-dependent opacity. The values of the "low-velocity" $\gamma \mathrm{s}$ for some young outflows, such as L1448 (data from Bachiller et al. 1990, plot from Bachiller \& Tafalla 1999) and NGC 2264G (Lada \& Fich 1996), were calculated from fits to the mass spectra only for velocities greater than $7.0 \mathrm{~km} \mathrm{~s}^{-1}$ from the line core, where the assumption that the line is optically thin is not a bad one. Thus, for those flows we would not expect the value of $\gamma$ to change significantly if a velocity-dependent opacity correction of the ${ }^{12} \mathrm{CO}$ line were included in the analysis. Ultimately, we expect that if all mass spectra were corrected for opacity, one would find a significant spread in the values of $\gamma$ perhaps even without a concentration of values around $\gamma \sim 2$.

There are a number of possible explanations for the relatively large value of $\gamma$ in $\mathrm{HH} 300$. In their paper, YBB present a list of factors that could modify the slope of the mass spectrum. We offer a similar list of factors here, but we focus on the elements that could give rise to a steep slope in HH 300.

YBB state that their outflow mass estimates are affected by the velocity at which they choose to define an optically thin limit for $R_{12 / 13}$, that is, the velocity at which $R_{12 / 13}$ is set to the carbon isotopic ratio (see $\S 3.3 .2$ and Fig. $7 a$ ). In our case, changing the isotopic ratio from 62 (the value assumed in this paper) to 89 (the solar ratio) changes the total mass of the red lobe of the HH 300 outflow by less than $1 \%$, as this change only affects the "high outflow velocity" (1.9 $\left.\mathrm{km} \mathrm{s}^{-1}<v<3.2 \mathrm{~km} \mathrm{~s}^{-1}\right)$ masses. Using any value for the isotopic ratio from 50 to 89 leaves the powerlaw slope of the low-velocity $\left(0.7 \mathrm{~km} \mathrm{~s}^{-1}<v<1.9 \mathrm{~km} \mathrm{~s}^{-1}\right)$ mass spectrum unchanged. The high-velocity $\gamma$ increases by a maximum of $4 \%$. Thus, the value of the carbon isotopic ratio does not affect our results considerably. (If we had used only a single power-law fit, the isotopic ratio would significantly effect the power-law slope, as in YBB.)

The outflow orientation with respect to the plane of the sky is another factor that can modify the mass spectrum slope. As shown in Smith et al. (1997), where molecular outflows are modeled as entrainment of ambient gas by a bow shock, the value of $\gamma$ increases as inclination angle (of the flow axis with respect to the plane of the sky) decreases. Specifically, the results of Smith et al. (1997) show one

TABLE 4

COMPARISON OF $\gamma$ FOR DIFFERENT VELOCITY-DEPENDENT OPACITY-CORRECTED OUTFLOWS

\begin{tabular}{|c|c|c|c|}
\hline \multirow[b]{2}{*}{ OUTFLOW } & \multicolumn{2}{|c|}{ AvERAGE $\gamma^{\mathrm{a}}$} & \multirow[b]{2}{*}{ REFERENCES } \\
\hline & Without Ambient Cloud Correction & With Ambient Cloud Subtracted & \\
\hline Circinus flow A...... & 4.0 & $\ldots{ }^{b}$ & 1 \\
\hline Circinus flow B...... & 3.7 & $\ldots{ }^{b}$ & 1 \\
\hline Circinus flow $\mathrm{C} \ldots \ldots$. & 3.8 & $\ldots{ }^{b}$ & 1 \\
\hline B5-IRS $1 \ldots \ldots \ldots \ldots$. & 3.9 & 2.7 & 2 \\
\hline MMS $9 \ldots \ldots \ldots \ldots . . . .$. & 3.8 & 1.8 & 3 \\
\hline 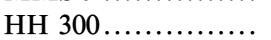 & 5.2 & 4.0 & 4 \\
\hline
\end{tabular}

${ }^{a}$ The number given is the average value of $\gamma$ over both lobes of the given outflow (except for HH 300, where only one lobe was studied). The value of $\gamma$ is defined to be the power-law slope in the relation $[d M(v) / d v] \propto v^{-\gamma}$. For the two outflows with mass spectra exhibiting power laws (HH 300 and MMS 9), we show the low-velocity $\gamma$.

${ }^{b}$ There is no mass spectrum with ambient cloud subtracted for these outflows.

REFERENCES.- (1) Bally et al. 1999. (2) Yu et al. 1999. (3) Yu et al. 2000. (4) This paper. 
model where decreasing the angle between the outflow axis and the plane of the sky from $60^{\circ}$ to $15^{\circ}$ raises $\gamma$ from $\sim 1.2$ to $\sim 1.5$. Although it is very probable that the axis of the HH 300 outflow is close to the plane of the sky $\left(i \lesssim 15^{\circ}\right)$, this would not be enough, by itself, to explain the high value of $\gamma$ $(\sim 4)$ in the red lobe of HH 300.

Contamination from cloud emission can also affect the slope of the mass spectrum. If one does not take the ambient cloud emission into consideration when estimating the outflow mass, one will overestimate the outflow mass at low velocities, and thus the mass spectrum slope will be overestimated. This was shown in $\S 3.3 .2$, where it can be seen that the value of $\gamma$ before correcting for the ambient cloud emission was larger before correction $(\gamma=5.2 \pm 0.1)$ than after it $(\gamma=4.0 \pm 0.2)$. On the other hand, our method for correcting the outflow mass for ambient cloud emission might slightly underestimate the mass from the lowest outflow velocity channels (as discussed in $\S 3.3 .3$ ). Underestimating the low-velocity outflow mass would result in an underestimation of the power-law slope. In order to check if we made a good correction, we constructed a mass spectrum of a limited area around clump R3. We made a powerlaw fit to only the channels between outflow velocities of 1.1 and $1.9 \mathrm{~km} \mathrm{~s}^{-1}$, which have predominantly outflow emission (based on the appearance of channel maps) and very little or no ambient cloud emission. The resulting mass spectrum, uncorrected for ambient cloud emission, has a $\gamma=3.9 \pm 0.2$, consistent with the mass spectrum of the whole outflow, corrected for ambient cloud emission, which is $\gamma=4.0 \pm 0.2$. Thus, we are convinced that our correction for ambient cloud emission is a good one and that the steep value of $\gamma$ that we obtain for the red lobe of the HH 300 outflow is not due to any residual ambient cloud emission at low velocities.

We believe that the most important factor in steepening the slope of the HH 300 mass spectrum is outflow evolution. Both the models of Smith et al. (1997) and Downes \& Ray (1999) find that the power spectrum slope of the mass spectrum changes over time. Both studies find that $\gamma$ steepens as the outflow ages. The ambient material that once was accelerated by bow shock entrainment slows down as time goes by, meaning more slow gas is accumulated, resulting in a steepening of $\gamma$. The simulated outflows of Smith et al. (1997) and Downes \& Ray (1999) are relatively young $(600$ and $300 \mathrm{yr}$, respectively), but their results seem to indicate that the increasing trend of $\gamma$ with age would continue as the outflow evolves in time.

Richer et al. (2000, hereafter RSCBC) do not find any indication of time evolution of the low-velocity $(v<10 \mathrm{~km}$ $\left.\mathrm{s}^{-1}\right) \gamma$ from outflow from low-luminosity $\left(L_{\mathrm{bol}}<10^{3} L_{\odot}\right)$ sources. ${ }^{2}$ The lack of a trend in their results might be explicable in two ways. First, RSCBC estimate the age of the outflows using their dynamical age. The dynamical age of an outflow is estimated using an average or typical velocity for the source being studied and dividing that velocity by the extent of the outflow lobe. This is known to be a poor

\footnotetext{
${ }^{2}$ YBSBB show a similar compilation of $\gamma$-values as a function of outflow dynamical age and as a function of outflow source luminosity. Their compilation is slightly larger than that of RSCBC and includes the B5-IRS 1 and MMS 9 outflows. Unlike RSCBC, YBSBB only use the steeper value of $\gamma$ (which in most outflows is the "high-velocity $\gamma$ ") for sources that have broken power laws. Similar to RSCBC, YBSBB's small sample makes it hard to state any definitive conclusion about outflow evolution.
}

estimate of the age of an outflow (it could be off by as much as a factor of 10-50) because it assumes that the outflow has kept the same "typical " velocity throughout its life and also because it assumes that all outflowing material comes from the immediate vicinity of the source (Masson \& Chernin 1993). Second, the outflow masses are obtained without correcting for a velocity-dependent opacity in the CO line, so there could be an underestimation of the value of $\gamma$ for some of the outflows in RSCBC's sample.

If our physical reasoning for the steepening of $\gamma$ is correct, we would then expect that $\gamma$ would evolve differently for outflows in physically different environments. Outflows in much denser mediums will decelerate more rapidly than outflows in low-density mediums if the entrainment mechanism is a momentum-conserving one, as it probably is (Masson \& Chernin 1993). It would be interesting to conduct a survey of outflows in similar environments, with mass corrected for $\mathrm{CO}$ velocity-dependent opacity and with accurate age estimates, in order to test how $\gamma$ evolves over time.

The analytical study by Matzner \& McKee (1999) find that it is hard to obtain a value of the power-law mass spectrum slope very different from 2. This value of $\gamma \sim 2$ is independent of the time history of the driving wind, including its momentum input as a function of time, and applies to a wide variety of ambient density distributions. The entrainment mechanism of the ambient medium in the Matzner \& McKee (1999) study does not specifically involve a bow shock. Instead, a collimated wind sweeps the ambient gas into a momentum-conserving shell, following the models of Shu et al. (1991) and Li \& Shu (1996). The Matzner \& McKee (1999) model does not take into account an episodic, precessing outflow that could be modifying the underlying density and velocity distribution of the surrounding gas with each mass ejection episode. As an episodic and precessing outflow changes direction, different mass outflow ejection episodes may be able to entrain new ambient material that previous ejections were not able to effect, as well as to reaccelerate gas that had already been put into motion by a previous ejection. These processes will certainly effect the mass spectrum slope of an outflow over time. More on this will be discussed in a subsequent paper (Arce \& Goodman 2001).

\subsection{The Episodic and Precessing Nature of the Flow}

The most striking thing about the morphology of the $\mathrm{HH}$ 300 outflow is its clumpy structure. The velocity maps in Figures 4 and 5 show discrete clumps in space and velocity. It is also notable that the outflow lobe axis does not have a fixed position angle in the plane of the sky. By drawing a line from the source to the maximum emission point for each of the five (redshifted) clumps in the outflow, we obtain position angles in the plane of the sky ranging from $250^{\circ}$ to $221^{\circ}$ (see Table 2). One unlikely interpretation of the flow's appearance is that the underlying wind that drives the outflow is a wide-angle wind, with an axis very close to the plane of the sky. In this interpretation most of the wind is not detected as the slow radial velocities are hidden under the ambient cloud emission. The redshifted clumps that we detect could then be where the wide-angle wind is interacting with preexisting cloud clumps, which have been moved to those redshifted velocities by the wind. This scenario seems untenable for several reasons. First, in a momentum-conserving wind-clump interaction, the lightest 
clumps should be the fastest moving clumps. This is not the case for the HH 300 clumps, where, for example, the heaviest clump (R2) has greater velocities than other, much lighter clumps. Second, if bow shock shapes are caused by the wind-clump interaction, one would expect them to have "downstream" wings. On the contrary, the several clumps that have bow shock-like structures have all wings pointing back to the source.

A more plausible explanation is that $\mathrm{HH} 300$ is a precessing and episodic outflow and that the outflow clumps are made of swept-up ambient gas. The density of the ambient gas in the B18w region should be on the order of $5 \times 10^{3}$ $\mathrm{cm}^{-3}$, and each outflow clump has a mass less than $0.5 M_{\odot}$ (see Table 2). In order to form a clump of about $0.5 M_{\odot}$ by sweeping up gas with density of $5 \times 10^{3} \mathrm{~cm}^{-3}$, a volume of about $4 \times 10^{52} \mathrm{~cm}^{3}$ is needed. A cube of side $0.12 \mathrm{pc}$ (or $3^{\prime}$ at a distance of $140 \mathrm{pc}$ ) is more than enough to account for such volume. From the integrated intensity maps (Figs. 4, 5, and 6), we see that the clumps have sizes comparable to or bigger than $0.12 \mathrm{pc}$. So it is realistic to say that most of the mass in the clumps could come from gas that has been accelerated very close to the current position of the clump. Another possible scenario is that the clumps formed (by swept-up ambient gas) at a position closer to IRAS $04239+2436$ and then moved to their current position (see $\S 4.5)$.
Position-velocity (PV) diagrams of $\mathrm{HH} 300$, constructed by summing all spectra along an axis with a position angle of $220^{\circ}$ (which hereafter will be called the outflow axis) are shown for the ${ }^{12} \mathrm{CO}(2-1)$ and ${ }^{12} \mathrm{CO}(1-0)$ lines in Figures 9 and 10. It can be seen that at the position of all the clumps except R5, there is an increase in the extreme emission velocity. The velocity structure at the position of each of these clumps is characteristic of the prompt entrainment mechanism, which will produce the highest velocities at the "hot spots" (or local CO maximum of clump), and decreasing velocity trend toward the source (Bence et al. 1996). The kinematic behavior where the velocity of an outflow increases nearly linearly with distance from the outflow source is referred to as the "Hubble law" for outflows. Thus, in our case, we can say that there is a different outflow Hubble law associated with each clump. For bow shock models (e.g., Zhang \& Zheng 1997; Smith et al. 1997; Downes \& Ray 1999), the Hubble law is a natural consequence of the fact that the highest velocities are found at the head of the bow shock, while the velocity decreases toward the wings. Wide-angle wind models of entrainment (Shu et al. 1991; Matzner \& McKee 1999) produce only a single Hubble-law velocity field. We can best explain the Hubblelaw "bumps" at the position of the clumps in Figures 9 and 10 as each being produced by a bow shock at the position of the clumps.

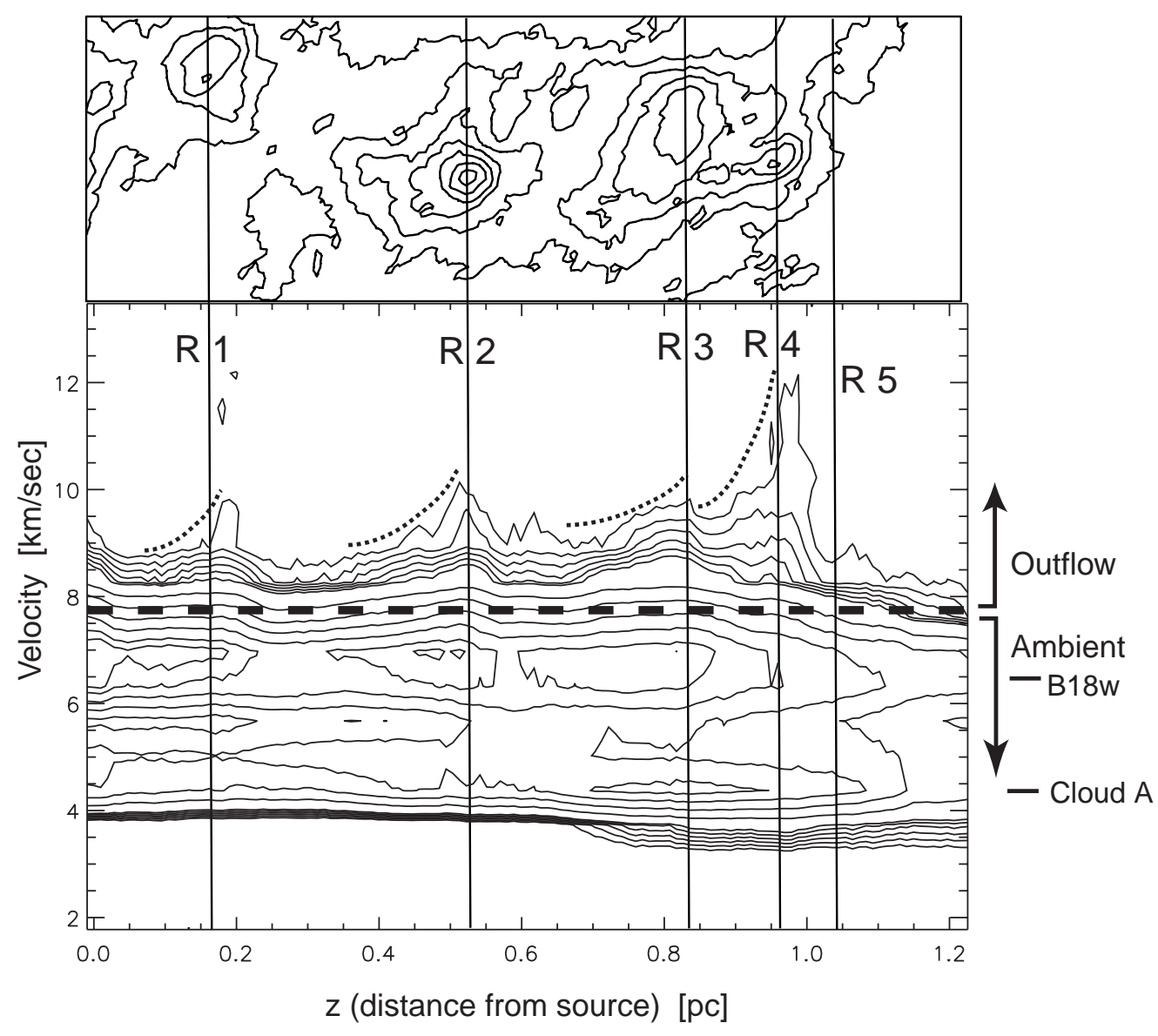

Fig. 9.- (Top) The ${ }^{12} \mathrm{CO}(2-1)$ integrated intensity contour map for velocities between 7.30 and $10.55 \mathrm{~km} \mathrm{~s}^{-1}$. (Bottom) The ${ }^{12} \mathrm{CO}(2-1)$ position-velocity diagram of the redshifted lobe of $\mathrm{HH} 300$. The outflow velocities, as well as the ambient gas velocities of the B18w cloud and of cloud A, are indicated on the right of the bottom panel. The position of each clump along the outflow axis is marked with a vertical solid line. Notice how at the position of each molecular outflow clump there is an increase in the velocity. The increase in velocity at most of the clump positions, as shown with the dotted curved lines, follows a Hubble law, where the velocity increases proportionally to the distance from the source. This velocity structure is consistent with the outflow clumps being produced by bow shock prompt entrainment from an underlying wind (see $\S 4.3$ for discussion). 


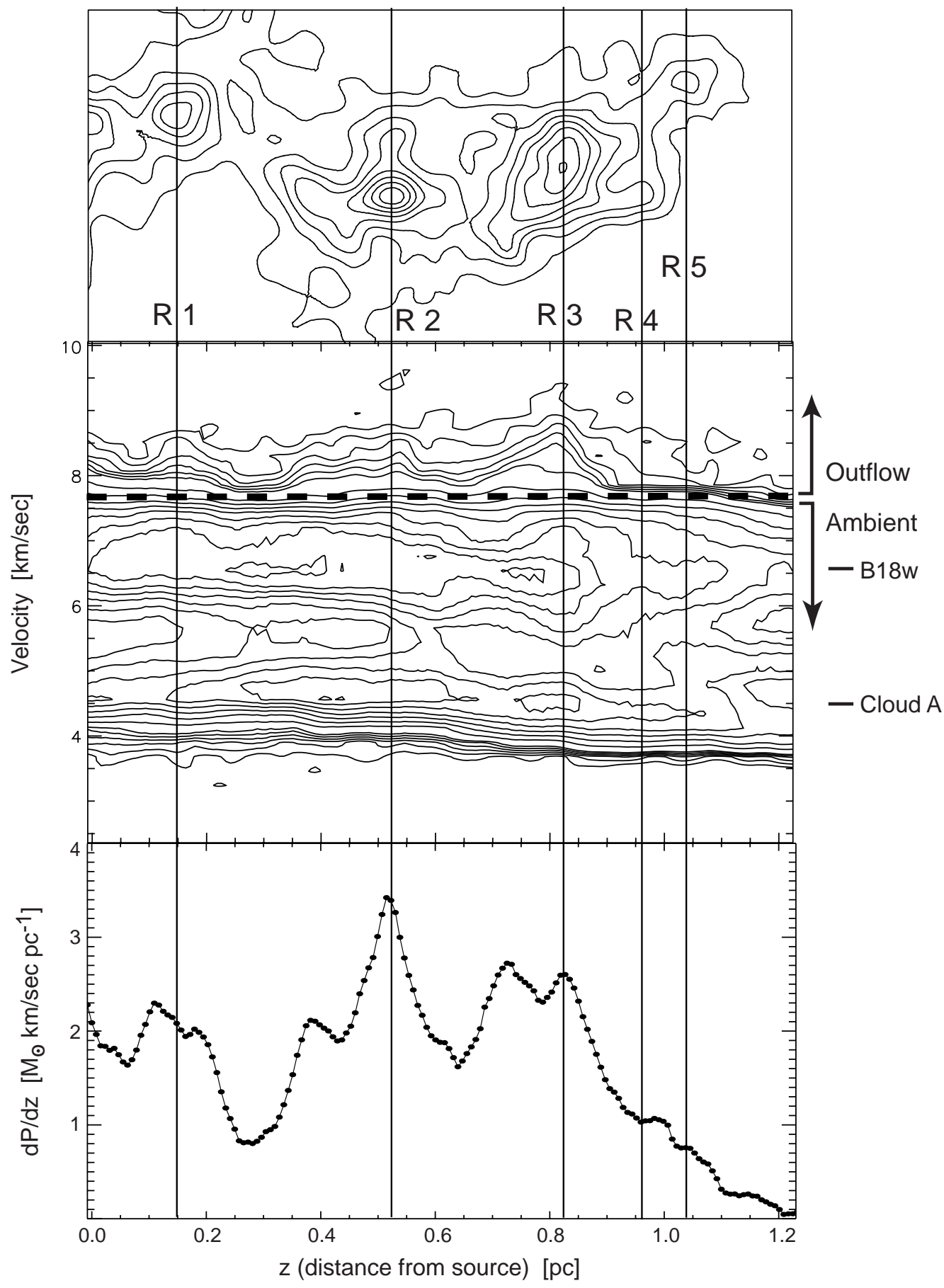

FIG. 10.-(Top) The ${ }^{12} \mathrm{CO}(1-0)$ integrated intensity contour map, as shown in Fig. 6. (Middle) The ${ }^{12} \mathrm{CO}(1-0)$ position-velocity diagram of the redshifted lobe of HH 300. (Bottom) Distribution of the outflow momentum per unit length $(d M / d z)$ along the HH 300 outflow axis. The filled circles represent the total momentum over the width of the outflow lobe. The outflow velocities, as well as the ambient gas velocities of the B18w cloud and of cloud A, are indicated on the right of the middle panel. The position of each clump along the outflow axis is marked with a vertical solid line. Notice that at the positions of the R1, R2, and $\mathrm{R} 3$ clumps, the three most prominent clumps in ${ }^{12} \mathrm{CO}(1-0)$, there is an increase in velocity and momentum (see $\S 4.3$ for discussion).

In the bottom panel of Figure 10, we show a plot of the total momentum per unit length (measured in cuts perpendicular to the outflow axis) as a function of distance from the source along the flow axis $(z)$, similar to what Chernin \& Masson (1995) do for several outflows. It is clear that at the position of each redshifted clump there is a peak in the momentum. The plot in the bottom panel of Figure 10 looks very similar to the plots produced by the precessing jet model of Cliffe, Frank, \& Jones (1996). In their models, each momentum peak corresponds to different shock structures in a precessing jet.

In $\mathrm{HH} 300$, the morphology of the knots in the outflow, the PV diagram, and the momentum distribution all support a picture where the outflow is made up of several bow shocks along its axis, where each shock corresponds to a different ejection event with a different ejection axis.

Recently, it has been established that it is common for the ejection axis of outflows to precess or wander (e.g., RBD). It 
is believed that outflow precession is due to the precession of the outflow source, which could itself be caused by the tidal interaction between the circumstellar disk of the outflow source and one (or many) stellar companion(s) of the source (e.g., Terquem et al. 1999). Reipurth et al. (2000) find that the source of the $\mathrm{HH} 300$ jet is a binary with a separation of only $42 \mathrm{AU}$, which could explain the precessing nature of the $\mathrm{HH} 300$ outflow. If we assume that clumps $\mathrm{R} 1$ and $\mathrm{R} 2$, the two clumps with the most extreme position angles, are close to the edges of the precession cone, then the precession period is approximately twice the difference in eruption age, $2\left(\tau_{\mathrm{dyn}, \mathrm{R} 2}-\tau_{\mathrm{dyn}, \mathrm{R} 1}\right) \approx 3400 \mathrm{yr}$ (see Table 2$)$. Terquem et al. (1999) give an approximate expression for the precession period of a disk around a young star in a binary system, where the disk surrounds only the primary star. The expression is given in terms of the primary mass, the mass ratio of the two stars, and the ratio of the disk radius to the binary orbit radius. If we assume a primary mass of $0.5 M_{\odot}$ and a primary-to-secondary mass ratio of about 1.6, the precession period is about 4000 yr. Hence, it is plausible that the precessing nature of the $\mathrm{HH} 300$ outflow could be explained by the fact that its source lies in a multiple-star system.

\subsection{Effects on the Cloud}

Although the HH 300 outflow is not impressively massive or energetic (like R Mon; Wolf, Lada, \& Bally 1990), it is still an "average" outflow (in the context of the outflow catalog of Wu, Huang, \& He 1996), which can apparently significantly effect the velocity structure of its host cloud. We have already shown that the $\mathrm{HH} 300$ flow is depositing a fair amount of momentum over a notable volume of its environment. Another way of assessing the effect that an outflow has on the parent cloud is by studying the behavior of the velocity dispersion as a function of position. By doing so we can examine the effects that the flow has on the cloud kinematics. To do this, we fitted a single Gaussian to the ${ }^{13} \mathrm{CO}(1-0)$ spectral component associated with B18w for all spectra southwest of the source. We then made a contour plot of the velocity width $(\Delta v)$ obtained through the Gaussian fit (Fig. 11). It appears that the outflow clumps are associated with peaks in the ${ }^{13} \mathrm{CO}(1-0)$ velocity width. Many of the ${ }^{13} \mathrm{CO}(1-0)$ spectra at the large- $\Delta v$ positions exhibit two peaks. We interpret these two separate velocity components as one arising from the cloud emission and one arising from both cloud and outflowing ${ }^{13} \mathrm{CO}(1-0)$ emission. The existence of these multiple peaks implies that in certain positions the outflow has put substantial amounts of gas in motion in order for the outflow spectra to appear as a separate velocity component from that of the cloud, rather than just a low-emission high-velocity wing in the cloud component. Hence, detecting one (or more) easily identifiable velocity components attributable to an outflow is yet another indication that the flow is having a major effect on the cloud gas kinematics.

Different velocity components in outflow spectra from high-density tracers have been observed in the Mon R2 outflow (Tafalla, Bachiller, \& Wright 1994). The clumps responsible for the multiple peaks in the CS spectra of the Mon R2 outflow have been interpreted as gas from the dense core that has been put into motion (Tafalla et al. 1997). On the other hand, the multiple velocity components arising from outflow ${ }^{12} \mathrm{CO}$ spectra observed in some lowand medium-mass stars (e.g., L1448, Bachiller et al. 1990;
IRAS 03282 + 3035, Bachiller, Martín-Pintado, \& Planesas 1991; HH 111, Cernicharo \& Reipurth 1996), usually called high-velocity bullets, have masses of the order of $10^{-3} M_{\odot}$ (e.g., Hatchell, Fuller, \& Ladd 1999) and are thought to be composed of jet material. The HH 300 redshifted clumps have relatively large masses $\left(\sim 0.1 M_{\odot}\right.$; see Table 2$)$, which makes it probable that they are mainly made up of sweptup material, more like the high-density clumps of the Mon $\mathrm{R} 2$ outflow. Therefore, we conclude that the ${ }^{13} \mathrm{CO}$ clumps in $\mathrm{HH} 300$ consist of medium-density $\left(n \sim 10^{3} \mathrm{~cm}^{-3}\right)$ gas entrained by the outflow.

We can quantify the effect that the $\mathrm{HH} 300$ flow has on its parent cloud using different methods. One method is to compare the flow's total energy with the cloud's total turbulent kinetic energy. The total kinetic energy of a cloud where thermal motions are a negligible part of $\Delta V$ is given by $E_{\text {turb }}=(3 / 16 \ln 2) M_{\text {cloud }} \Delta V^{2}$, where $M_{\text {cloud }}$ is the cloud mass and $\Delta V$ is the observed FWHM velocity line width. In our case, from the ${ }^{13} \mathrm{CO}(1-0)$ data, the mass of the whole B18w filamentary structure (seen in Fig. $3 b$ ) is $M_{\text {cloud }}=72$ $M_{\odot}$. The velocity width is $\Delta V \sim 0.8 \mathrm{~km} \mathrm{~s}^{-1}$, so $E_{\mathrm{turb}} \sim 3$ $\times 10^{44}$ ergs. The outflow kinetic energy is about $2.6(\sin i)^{-2} \times 10^{43}$ ergs; thus, the HH 300 outflow has the potential of making a substantial contribution to the turbulent energy of the cloud. For $i=17^{\circ}$, the outflow kinetic energy is equal to the kinetic energy of the $\mathrm{B} 18 \mathrm{w}$ region. Armstrong \& Winnewisser (1989) discovered that the kinetic energy of a parsec-scale outflow in L673 could be 6-0.4 times (depending on the assumed projection angle and the amount of mass hidden under the line core) the turbulent energy in the cloud. At the time Armstrong \& Winnewisser (1989) made their study, astronomers had no idea of the common occurrence of parsec-scale $\mathrm{HH}$ flows from young stellar objects. Now, with the discovery that $\mathrm{HH}$ flows may typically extend for several parsecs (RBD), we should reconsider the role of outflows in driving the turbulent energy of molecular clouds.

We can also asses the importance of the HH 300 outflow energy input on its parent cloud by using the results of numerical simulations. The self-consistent magnetohydrodynamic (MHD) simulations of Gammie \& Ostriker (1996) study MHD turbulence under density, temperature, and magnetic field conditions representative of those found in Galactic molecular clouds. The models use "slab symmetry," in which all variables are a function of one independent spatial variable $x$ and time $t$. Using equation (34) in Gammie \& Ostriker (1996), we can derive the input power needed to support a molecular cloud in equilibrium. We approximate the "linear" dimension $(L)$ of B18w to be twice the geometric mean of its axes $(\sim 1.1 \mathrm{pc})$. We assume a mean density of $5 \times 10^{3} \mathrm{~cm}^{-3}$ and a $\beta$-parameter ${ }^{3}$ of 0.01 . Using these approximations we find that we would need about $0.4 L_{\odot}$ of input power at a scale of $1 \mathrm{pc}$ (the size of the outflow lobe) to counter the dissipation of MHD turbulence in the B18w region and thus support it against gradual gravitational contraction.

The power of an outflow is usually estimated by dividing the outflow kinetic energy by the dynamical age of the outflow. The conventional way to estimate the dynamical age of a molecular outflow assumes that all the gas in the outflow originates at the young star. This assumption is

\footnotetext{
${ }^{3}$ The $\beta$-parameter is defined as the ratio of the square of the sound speed to the square of the Alfvén speed in the cloud; that is, $\beta=c_{s}^{2} / v_{\mathbf{A}}^{2}$.
} 


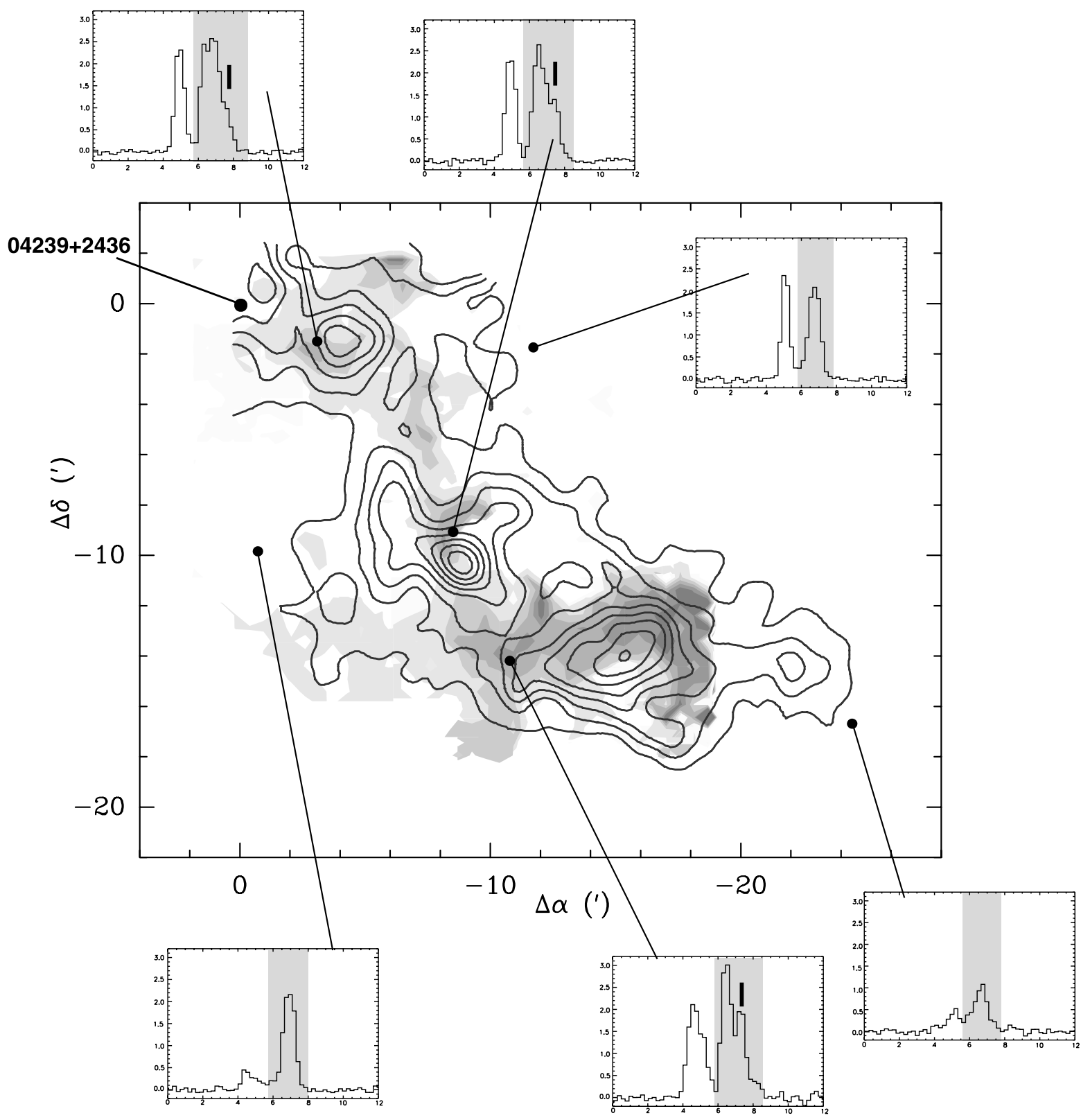

FIG. 11.- The ${ }^{12} \mathrm{CO}(1-0)$ integrated intensity map of the $\mathrm{HH} 300$ outflow, as in Fig. 6, superimposed on a gray-scale map of ${ }^{13} \mathrm{CO}(1-0)$ line width (FWHM). First gray tone corresponds to a width of $1.0 \mathrm{~km} \mathrm{~s}^{-1}$. Between each gray tone there is an increment of $0.2 \mathrm{~km} \mathrm{~s}^{-1}$. Coordinate offsets are given with respect to the outflow source's position at $(\alpha, \delta)_{1950}=\left(4^{\mathrm{h}} 23^{\mathrm{m}} 54^{\mathrm{s}} .5,24^{\circ} 36^{\prime} 54^{\prime \prime}\right)$. Six example ${ }^{13} \mathrm{CO}(1-0)$ spectra at different position are shown. In all spectra, the cloud A and the B18w spectral components can be seen. The B18w (+ HH 300 in some cases) spectral component is highlighted in gray. Three spectra are from regions presumably unaffected by the HH 300 outflow, and three are from regions of the cloud affected by the outflow. On these last three, a vertical solid line indicates the position of the separate spectral component, presumably due to the outflow. All spectra are plotted with the same axes ranges: 0 to 12 $\mathrm{km} \mathrm{s}^{-1}$ in the velocity axis and -0.2 to $3.2 \mathrm{~K}$ on the antenna temperature axis.

wrong, since the vast majority of the gas in a molecular outflow comes from the entrained gas in the host cloud (along the extent of the outflow) that has been put in motion by the underlying stellar wind. Since there is no way to obtain an accurate estimate of the outflow lifetime, we estimate a lower and an upper bound to the dynamical age in order to estimate an upper and lower bound to the outflow power. We estimate the dynamical age lower limit to be the time it has taken $\mathrm{HH} 300 \mathrm{~A}$ (the $\mathrm{HH}$ knot that currently lies further to the source) to travel to its current position. The distance from the source to $\mathrm{HH} 300 \mathrm{~A}$ is $1.2 \mathrm{pc}$, and if we assume a typical $\mathrm{HH}$ jet velocity of $200 \mathrm{~km} \mathrm{~s}^{-1}$, we then obtain a dynamical age of about $5900 \mathrm{yr}$. As an upper limit on the age, we use $2 \times 10^{5} \mathrm{yr}$, the statistical lifetime of outflows as derived by the study of Parker, Padman, \& Scott (1991). We then estimate the lower and upper limits of the outflow power to be $1.1 \times 10^{-3}(\sin i)^{-2}$ to $3.6 \times 10^{-2}(\sin i)^{-2} L_{\odot}$. If we assume that $i \sim 10^{\circ}$, then the outflow power's lower limit is only about a factor of 10 less than what is needed to drive the MHD turbulence in B18w. Thus, the HH 300 outflow has the potential to make a substantial contribution to the power needed to sustain the MHD turbulence in B18w.

Yet another way to estimate the effects of the flow on the cloud is to compare the flow's energy with the cloud's binding energy. The B18w cloud binding energy 
( $\left.\sim G M_{\text {cloud }}^{2} / R\right)$, where we estimate $R$ to be the geometric mean of the short and long axes of B18w, is about $8 \times 10^{44}$ ergs. Hence, if $i \sim 10^{\circ}$, the HH 300 outflow would have enough kinetic energy to potentially disperse a major fraction of the B18w region. From our estimates (see $\S 4.1$ ), we see that $\mathrm{HH} 300$ is effecting a notable fraction of $\mathrm{B} 18 \mathrm{w}$, so whether most of the outflow kinetic energy will be converted to turbulent energy or used to directly disperse the cloud gas (see $\S 4.6$ for more on this), it is clear that the $\mathrm{HH}$ 300 outflow will have a major effect on the evolution of B18w.

\subsection{The Structure of $B 18 w$}

The axis of the $\mathrm{HH} 300$ redshifted outflow lobe is parallel to B18w's long axis, as can be seen in both Figures 2 and 6 . The B18w region looks like a protuberance that sticks out from the main B18 molecular cloud (see Fig. 1). Given the nice positional coincidence of the outflow's and the dark cloud's axes, in addition to the particular shape of B18w and its position with respect to the rest of the B18 cloud, we originally flirted with the idea that the $\mathrm{HH} 300$ outflow was creating the $\mathrm{B} 18 \mathrm{w}$ dark cloud. We hypothesized that the outflow was dragging gas and dust from the star-forming core and forming the B18w dark cloud. Without calculating any estimates of mass for $\mathrm{HH} 300$ and $\mathrm{B} 18 \mathrm{w}$, this seemed like a reasonable idea. Similarly, Bence et al. (1996) speculate that the RNO 43 outflow's most southerly bow shock could be responsible for ejecting material from the main cloud and creating a spur of cloud material, seen in ${ }^{12} \mathrm{CO}$ emission and in extinction (see their Fig. 19). But, once we estimated the mass of the outflowing material to be only $7 \%$ that of the surrounding ambient gas, in addition to studying the kinematics of the gas, we concluded that what we observe to be the "current" HH 300 molecular outflow has not created the $\mathrm{B} 18 \mathrm{w}$ region. Moreover, if this scenario were true, one would expect the blue and the red lobes to drag along similar amounts of gas. As seen in Figures 1 and 8, the B18w cloud extends southwest with respect to the outflow source but does not extend northeast of the source, where one would expect the blue lobe to be. Such a high asymmetry in the "dragging process" is highly unlikely. Even if this scenario does not apply to the HH 300 outflow, we still believe that the process in which an outflow ejects and/or drags gas and dust from a dense core (or any other dense region in the parent cloud) and moves it to distances of the order of $0.1-1 \mathrm{pc}$ could take place in other sources. If $\mathrm{HH}$ 300 is not "producing" the B18w region, then the fact that $\mathrm{B} 18 \mathrm{w}$ and $\mathrm{HH} 300$ have long axes with similar position angles is a coincidence. In that case, HH 300 has a shape similar to B18w because B18w is the cloud that "supplies" the gas that HH 300 is made of. That is, the parent cloud is constraining the morphology of the observed outflow.

\subsection{The Cumulative Effect of Outflows}

Our observations clearly show that even low-mass young stellar objects with a luminosity of about $1 L_{\odot}$ can affect their surrounding density and kinematical distribution on parsec scales. From our results, we can speculate that the cumulative action of many outflows from different YSOs could have a profound effect on a cloud's evolution and fate. The cumulative effect of many outflows could be (1) to produce just enough turbulent energy to support the cloud against gravitational collapse, (2) to greatly increase the turbulent energy to more than the binding energy of the cloud, or (3) to put enough ambient gas into motion as to directly disperse the cloud. If enough gas is moved out of the gravitational potential well of the cloud, the cloud would eventually disperse. If the ambient density is relatively high and the outflow momentum is relatively low, then the outflowing material will eventually slow down through the interaction with the ambient medium, mixing with its surroundings and feeding the turbulent energy of the cloud. On the other hand, if the ambient density is relatively low and the outflow momentum is high, the swept-up gas will barely decelerate and it could eventually be "pushed away" from the cloud. It is hard to conclude which of these two scenarios will prevail in a given cloud without modeling the interface between outflow and cloud. Further theoretical and numerical investigations should concentrate on how efficiently bulk motions, produced by outflows, can decay to produce turbulence as a function of a cloud's density and outflow energy. Now that it has been established that individual outflows are energetic enough and large enough to substantially affect a big portion of their host cloud, observational studies should concentrate in investigating the cumulative effects that many outflows have on a molecular cloud and on a cloud complex (e.g., see BRLB).

\section{CONCLUSION}

We mapped the red lobe of the giant molecular outflow associated with the $\mathrm{HH} 300$ flow in the ${ }^{12} \mathrm{CO}(2-1)$ line, with a beam size of $27^{\prime \prime}$. We also made a more extended map of the gas surrounding the $\mathrm{HH} 300$ flow at the ${ }^{12} \mathrm{CO}(1-0)$ and ${ }^{13} \mathrm{CO}(1-0)$ lines, with $45^{\prime \prime}$ and $47^{\prime \prime}$ beam sizes, respectively. By observing a large extent of the gas surrounding the outflow, we are able to study the outflow in the context of its surrounding medium. Also, the ${ }^{13} \mathrm{CO}(1-0)$ observations help us assess the effects the outflow has on the surrounding moderate-density $\left(n \sim 10^{3} \mathrm{~cm}^{-3}\right)$ gas structure and kinematics. In addition, the combined ${ }^{12} \mathrm{CO}(1-0)$ and ${ }^{13} \mathrm{CO}$ (1-0) line observations enable us to estimate the mass of the outflow by correcting for the velocity-dependent opacity of the ${ }^{12} \mathrm{CO}(1-0)$ line. Due to contamination from emission of another molecular cloud along the same line of sight, we are not able to study the blue lobe of the HH 300 outflow.

Our results show that the $\mathrm{HH} 300$ outflow has a very clumpy structure. We identify five ${ }^{12} \mathrm{CO}$ redshifted clumps, each of which is readily apparent in position-position-velocity space. They each have masses of the order of a few $10^{-1}$ $M_{\odot}$ and reach radial velocities of about $3 \mathrm{~km} \mathrm{~s}^{-1}$ from the ambient cloud velocity. Given the low inclination of the outflow with respect to the plane of the sky, the deprojected velocities of these clumps are likely to be higher than $15 \mathrm{~km}$ $\mathrm{s}^{-1}$. Such high masses and low velocities suggest that these clumps are made of swept-up ambient gas. The spatial distribution of the clumps, the velocity structure, and the momentum distribution of the outflow indicate that these clumps arise from prompt entrainment, most probably produced by bow shocks, arising from the young star's mass ejection episodes. Each of these clumps has a different orientation on the plane of the sky (or position angle) with respect to the outflow source. We conclude that $\mathrm{HH} 300$ is a precessing and episodic outflow.

We obtain a power-law mass spectrum slope of $-4.0 \pm 0.2$ for low outflow velocities and a slope of $-7.8 \pm 0.4$ for high outflow velocities for the red lobe of $\mathrm{HH} 300$. These slopes are steeper than the average for other 
outflows. To obtain the outflow mass, we used a velocitydependent ${ }^{12} \mathrm{CO}(1-0)$ optical depth, and we corrected the low-velocity outflow mass for contaminating ambient cloud emission. Previous outflow studies, with the exception of Bally et al. (1999), Yu et al. (1999), and Yu et al. (2000), from which we obtain the idea, do not correct for the velocitydependent opacity of the line when obtaining outflow parameters from their ${ }^{12} \mathrm{CO}$ data. As we have shown, not applying this correction will underestimate the low-velocity outflow mass. We believe that some low-velocity outflows will exhibit a steeper mass spectrum power-law slope $(\gamma)$ once their masses have been corrected for the velocitydependence of the ${ }^{12} \mathrm{CO}$ line. Thus, we expect that for a sample of outflows (with different ages), the value of $\gamma$ will have a wider range of values than what is currently found in the literature. The fact that $\mathrm{HH} 300$ has a steep mass spectrum power-law slope $(\gamma \sim 4)$ is most probably due to the evolution of outflow mass kinematics. Ambient material accelerated by several episodes of mass ejection by the young star will eventually slow down, leading to accumulation of slow gas and a steepening of $\gamma$.

The HH 300 outflow, although not extremely powerful, is depositing a fair amount of momentum $\left[3.2(\sin i)^{-1} M_{\odot}\right.$ $\left.\mathrm{km} \mathrm{s}^{-1}\right]$ and kinetic energy $\left[2.6(\sin i)^{-2} \times 10^{43} \mathrm{ergs}\right]$ over a notable volume $(\sim 11 \%)$ of its parent dark cloud (B18w). We qualify the effects that the $\mathrm{HH} 300$ flow has on its host cloud by using three different methods. If $\mathrm{HH} 300$ has an inclination to the plane of the sky (i) of about $10^{\circ}$, then the red lobe of the HH 300 molecular outflow has more than enough energy to supply the turbulent energy of B18w, more than enough energy to gravitationally unbind the B18w region, and enough power to make a substantial contribution to the power needed to sustain the MHD turbulence in $\mathrm{B} 18 \mathrm{w}$. In addition, the ${ }^{13} \mathrm{CO}$ data indicate that the HH 300 flow is having a major effect on the cloud gas kinematics and is able to redistribute considerable amounts of its surrounding medium-density $\left(\sim 10^{3} \mathrm{~cm}^{-3}\right)$ gas.

Our study clearly shows that even low-mass young stellar objects of about $1 L_{\odot}$ can produce drastic changes in their surrounding environment and affect a notable volume of their parent clouds. This is probably a characteristic of giant $\mathrm{HH}$ flows, which typically extend over a few parsecs on the sky and precess. Giant outflows have the potential of modifying the velocity and density structures not only of their host core but also of their host cloud, even at parsecscale distances from the source. The cumulative action of giant outflows from young stellar objects will certainly have a profound effect on a cloud's evolution and fate.

We are grateful to Bo Reipurth, John Bally, and Ka Chun $\mathrm{Yu}$ for their useful comments and to the National Science Foundation (AST 94-57456 and AST 97-21455) for supporting our efforts.
Arce, H. G., \& Goodman, A. A. 2001, ApJ, 551, L171

Armstrong, J. T., \& Winnewisser, G. 1989, A\&A, 210, 373

Bachiller, R., Cernicharo, J., Martín-Pintado, J., Tafalla, M., \& Lazareff, B. 1990, A\&\&A, 231, 174

Bachiller, R., Martín-Pintado, J., \& Planesas, P. 1991, A\&A, 251, 639

Bachiller, R., \& Tafalla, M. 1999, in The Origin of Stars and Planetary Systems, ed. C. J. Lada \& N. D. Kylafis (NATO ASI Ser. C, 540; Dordrecht: Kluwer), 227

Bally, J., Reipurth, B., Lada, C. J., \& Billawala, Y. 1999, AJ, 117, 410 (BRLB)

Bence, S. J., Richer, J. S., \& Padman, R. 1996, MNRAS, 279, 866

Bourke, T. L., et al. 1997, ApJ, 476, 781

Cabrit, S., Goldsmith, P. F., \& Snell, R. L. 1988, ApJ, 334, 196

Cernicharo, J., \& Reipurth, B. 1996, ApJ, 460, L57

Chernin, L. M., \& Masson, C. R. 1995, ApJ, 455, 182

Cliffe, J. A., Frank, A., \& Jones, T. W. 1996, MNRAS, 282, 1114

Davis, C. J., Eislöffel, J., Ray, T. P., \& Jenness, T. 1997, A\&A, 324, 1013

Downes, T. P., \& Ray, T. P. 1999, A\&A, 345, 977

Estalella, R., \& Anglada, G. 1997, Introducción a la Física del Medio Interestelar (1a ed.; Barcelona: Edicions Universitat de Barcelona)

Frerking, M. A., Langer, W. D., \& Wilson, R. W. 1982, ApJ, 262, 590

Gammie, C. F., \& Ostriker, E. C. 1996, ApJ, 466, 814

Greene, T. P., \& Lada, C. L. 1996, ApJ, 461, 345

Hatchell, J., Fuller, G. A., \& Ladd, E. F. 1999, A\&A, 346, 278

Kenyon, S. J., Dobrzycka, D., \& Hartmann, L. 1994, AJ, 108, 1872

Kutner, M. L., \& Ulich, B. L. 1981, ApJ, 250, 341

Lada, C. J., \& Fich, M. 1996, ApJ, 459, 638

Langer, W. D., \& Penzias, A. A. 1993, ApJ, 408, 539

Lee, C.-F., Mundy, L. M., Reipurth, B., Ostriker, E. C., \& Stone, J. M. 2000, ApJ, 542, 925

Li, Z.-Y., \& Shu, F. H. 1996, ApJ, 472, 211

Liseau, R., \& Sandell, G. 1986, ApJ, 304, 459

Masson, C. R., \& Chernin, L. M. 1993, ApJ, 414, 230

\section{REFERENCES}

Matzner, C. D., \& McKee, C. F. 1999, ApJ, 526, L109

Matzner, C. D., \& McKee, C. F. 2000, ApJ, 545, 364

Mizuno, A., Onishi, T., Yonekura, Y., Nagahama, T., Ogawa, H., \& Fukui, Y. 1995, ApJ, 445, L161

Moriarty-Schieven, G. H., Wannier, P. G., Tamura, M., \& Keene, J. 1992, ApJ, 400, 260

Myers, P. C., Fuller, G. A., Mathieu, R. D., Beichman, C. A., Benson, P. J., Schild, R. E., \& Emerson, J. P. 1987, ApJ, 319, 340

Norman, C., \& Silk, J. 1980, ApJ, 238, 158

Parker, N. D., Padman, R., \& Scott, P. F. 1991, MNRAS, 252, 442

Raga, A. C., Cantó, J., \& Steffen, W. 1996, QJRAS, 37, 493

Reipurth, B., Bally, J., \& Devine, D. 1997, AJ, 114, 2708 (RBD)

Reipurth, B., Yu, K. C., Heathcote, S., Bally, J., \& Rodríguez, L. F. 2000, AJ, 120, 1449

Richer, J. S., Shepherd, D. S., Cabrit, S., Bachiller, R., \& Churchwell, E. 2000 , in Protostars and Planets IV, ed. V. Mannings, A. P. Boss, \& S. S. Russell (Tucson: Univ. Arizona Press), 867 (RSCBC)

Rohlfs, K., \& Wilson, T. L. 1996, Tools of Radio Astronomy (2d ed.; New York: Springer)

Shu, F. H., Ruden, S. P., Lada, C. J., \& Lizano, S. 1991, ApJ, 370, L31

Smith, M. D., Suttner, G., \& Yorke, H. W. 1997, A\&A, 323, 223

Tafalla, M., Bachiller, R., \& Wright, M. C. H. 1994, ApJ, 432, L127

Tafalla, M., Bachiller, R., Wright, M. C. H., \& Welch, W. J. 1997, ApJ, 474, 329

Terquem, C., Eislöffel, J., Papaloizou, C. B., \& Nelson, R. P. 1999, ApJ, 512, L131

Wolf, G. A., Lada, C. J., \& Bally, J. 1990, AJ, 100, 1892

Wood, D., Myers, P. C., \& Daugherty, D. A. 1994, ApJS, 95, 457

Wu, Y., Huang, M., \& He, J. 1996, A\&AS, 115, 283

Yu, K. C., Billawala, Y., \& Bally, J. 1999, AJ, 118, 2940 (YBB)

Yu, K. C., Billawala, Y., Smith, M. D., Bally, J., \& Butner, H. 2000, AJ, 120, 1974 (YBSBB)

Zhang, Q., \& Zheng, X. 1997, ApJ, 474, 719 